\title{
Class formations III
}

\author{
By Yukiyosi KAWADA
}

(Received Nov. 25, 1955)

This paper is a continuation of our former papers (Kawada [7], Kawada-Tate [8]) concerning class formations and their application in algebraic function fields in one variable. After some preliminaries in $\S 1$ we shall consider in $\S 2$ the system of groups $\{W(K / k)\}$ in a class formation, which was investigated by A. Weil [12] in case of number fields. We shall arrange the formulas for $\{W(K / k)\}$ so that we are able to consider their inverse limit groups in $\S 3$. The sections $\S 4, \S 5$ are devoted to the application of the results in $\S 2$, $\S 3$ to the case of algebraic function fields. There we shall find the explicit structure of these groups $\{W(K / k)\}$ and their limit groups using the formulations in [8].

The results of A. Weil [12] were treated cohomology-theoretically first by T. Nakayama and G. Hochschild [5], [10]. Though the results in $\S 2$, $\S 3$ of this paper are not published hitherto in the literature, they would be known by mathematicians working in this field. The author does not claim any priority on these results. It should be mentioned that there are unpublished investigations of E. Artin and J. Tate concerning the structure of the inverse limit groups of $\{W(K / k)\}$ in case of number fields. Also the explicit structure of $\{W(k)\}$ in Theorem 6 (§5) was suggested by J. Tate. The author wishes to express his hearty thanks to Professor John Tate for his discussions during the preparation of this paper.

\section{$\S$ 1. Preliminaries}

1. We repeat here some necessary preparations from Part I (Kawada [7]) which we need later. Let $k_{0}$ be a fixed ground field and $\Omega$ be a fixed infinite separable normal algebraic extension of $k_{0}$. Let $\Omega$ be the set of all finite extensions of $k_{0}$ which are contained 
in $\Omega$. In the following we denote the fields which belong to $\Re$ by $k, l, K, L$ etc.

We assume further that a certain abelian group $A(K)$ is associated with each $K \in \Omega$ with the following properties: F1. Let $k \subset K$ then there exists an isomorphism $\varphi_{K, k}$ of $A(k)$ into $A(K)$. F 2 . Let $k \subset \boldsymbol{l} \subset K$ then the transitivity relation $\boldsymbol{\varphi}_{K, l} \circ \boldsymbol{\varphi}_{l, k}=\boldsymbol{\varphi}_{K, k}$ holds. $\mathrm{F} 3$. Let $K / k$ be a normal extension with the Galois group $G=G(K / k)^{1)}$ then $G$ acts on $A(K)$ as an operator group such that $\varphi_{K, k} A(k)=A(K)^{G}$ holds. ${ }^{2)}$ F 4. In

CASE I: $k \subset K \subset L, K / k$ and $L / k$ are both normal with the Galois groups $G=G(L / k), H=G(L / K)$ and $F=G(K / k)$

the relation $\sigma \circ \boldsymbol{\varphi}_{L, K}(a)=\boldsymbol{\varphi}_{L, K} \circ\left(\psi_{F, G} \sigma\right)(a)^{3)}$ holds for every $\sigma \in G$ and $a \in A(K)$. Furthermore, for every normal extension $K / k$ with $G G(K / k)$ we assume C 1: $\quad H^{1}(G, A(K))=0$ and $\mathrm{C} 2: \quad H^{2}(G, A(K))$ $\cong Z /[G: 1] \cdot Z$. We call then $\{\Re, A(K)\}$ a class formation.

Let $\{\Re, A(K)\}$ be a class formation. Then we can choose for each normal extension $K / k$ a 2-cocycle $f_{K / k}$ of $G$ over $A(K)$ with the following properties: (i) the cohomology class of $f_{K / k}$ is a generic class of $H^{2}(G, A(K))$, (ii) in case I we have $\operatorname{infl}_{G, F} f_{K / k} \sim f_{L / k}^{m}(m=[L: K])$, (iii) in

CASE II: $k \subset l \subset K, K / k$ is normal with $G=G(K / k)$ and $H=G(K / \mathfrak{l})$ we have $\operatorname{res}_{H, G} f_{K l k} \sim f_{K l l}{ }^{1)}$ We call then that $\left\{f_{K / k}\right\}$ is a system of fundamental (canonical) 2-cocycles in this class formation.

Let $\left\{g_{K / k}\right\}$ be another system of 2-cocycles. Then $\left\{g_{K / k}\right\}$ is also a system of fundamental 2-cocycles if and only if (i) $g_{K / k} \sim f_{K / k}^{r_{K l k}}, \boldsymbol{r}_{K / k}$ and $[K: k]$ are relatively prime; (ii) in case $\mathrm{I} \boldsymbol{r}_{L / k} \equiv r_{K / k}(\bmod [K: k])$; (iii) in case II $r_{K / l} \equiv r_{K / k}(\bmod [K: \imath])$.

Now let us consider the group pairing $(Z, A) \rightarrow A$ by $(n, a) \rightarrow a^{n}$.

1) We use the notation $G(K / k)$ to denote the Galois group of a normal extension $K / k$.

2) In a $G$-group $A$ we assume $a^{\sigma \tau}=\left(a^{\tau}\right)^{\sigma}$. By $A G$ we mean the set of all elements $a \in A$ which are invariant by all $\sigma \in G$.

3) For a subgroup $H$ of $G$ we mean by ${ }^{\imath} G, H$ the injection mapping $H \rightarrow G$ and for a normal subgroup $H$ of $G$ and $F=G / H$ we mean by $\psi_{F, G}$ the canonical homomorphism $G \rightarrow F$. As usual we mean by $Z, R$ and $C$ the modules of all integers, all real numbers and all complex numbers respectively.

4) By res $H, G$ we mean the restriction mapping: $H^{r}(G, A) \rightarrow H^{r}(H, A)$ for an abelian $G$-group $A$ in case II, and by $\operatorname{infl}_{G, F}$ we mean the inflation mapping (lift): $H^{r}(F, A H) \rightarrow$ $H^{r}(G, A)$ in case $\mathrm{I}$. 
Then the cup-multiplication $g^{r} \rightarrow g^{r} \cup f_{K / k}$ gives the isomorphism: $H^{r}(G, Z) \cong H^{r+2}(G, A(K))$ for all $r \in Z$ (Theorem of Tate [11]). In particular, for $r=-2 H^{-2}(G, Z) \cong G / G^{c}$ is isomorphic to $H^{0}(G, A(K))$ $\cong A(K)^{G} / N_{G} A(K)^{j)}$ by the mapping $\sigma(\bmod G) \rightarrow f_{K / k}(G, \sigma) \quad(\bmod$ $\left.N_{G} A(K)\right)^{6)}$ (Isomorphism theorem). We define the norm-residue symbol $(a, K / k)=\sigma\left(\bmod G^{c}\right)$ for $\varphi_{K, k}(a) \equiv f_{K / k}(G, \sigma)\left(\bmod N_{G} A(K)\right)$.

Let $A_{k}$ be the maximal abelian extension of $k$ in $\Omega$ and we denote by $\Gamma^{\top}(k)$ the compact Galois group $G\left(A_{k} / k\right)$. The generalized norm-residue symbol $(a, k)(a \in A(k))$ which takes value in $\Gamma(k)$ is defined as the limit of $\left(a, K_{\lambda} / k\right)$ for $k \subset K_{\lambda} \subset A_{k^{\circ}}$ The definition $(a, k)$ depends on the choice of a system of fundamental 2-cocycles $\left\{f_{K / k}\right\}$. But if we define $(a, k)^{\prime}$ by menas of another system $\left\{g_{K / k}\right\}$, then there exists an isomorphism $\Phi$ of $\Gamma^{\prime}(k)$ onto itself such that $(a, k)^{\prime}=$ $\Phi \circ(a, k)$ holds for all $a \in A(k)$.

The following two axioms are satisfied in case of class field theory and in several other cases of class formations.

P. For any extension $K / k(k, K \in \Re)$ there exists a cyclic extension $Z / k$ such that $[Z: k]$ is a multiple of $[K: k]$.

$\mathrm{P}^{\prime}$. There exists an increasing sequence of fields $K_{1}, K_{2}, \cdots, K_{n}, \cdots$ in $\AA$ such that $\Omega=\bigcup_{n=1}^{\infty} K_{n}$ holds.

LEMMA 1. Suppose that the axiom $\mathrm{P}$ is satisfied in $\Omega$. If the generalized norm-residue symbols $(a, k)(a \in A(k))$ defined by two systems $\left\{f_{K / k}\right\}$ and $\left\{g_{K / k}\right\}$ of fundamental 2-cocycles coincide, then $f_{K / k} \sim g_{K / k}$ holds for every normal $K / k$.

(PROOF) Let $g_{K / k} \sim f_{K / k}^{r} K_{k}$ then we have $(a, K / k)^{r} K / k=(a, K / k)$ by our assumption. Especially this implies $r_{Z l k} \equiv 1(\bmod [Z: k])$ for cyclic extension $Z / k$. Now for an arbitrary extension $K / k$ take a cyclic extension $Z / k$ such that $[Z: k]$ is a multiple of $[K: k]$. Then from the relations $r_{K Z \mid k} \equiv r_{Z / k}(\bmod [Z: k])$ and $r_{K Z \mid k} \equiv r_{K / k}(\bmod [K: k])$ follows that $r_{K / k} \equiv 1(\bmod [K: k])$. This means $f_{K / k} \sim g_{K / k}$, q. e.d.

2. We remark here some elementary properties of group extensions. Let $A, E, G$ be groups, ८, $\psi$ be homomorphisms such that

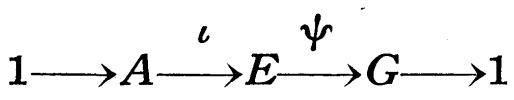

5) $G c$ means the commutator group of $G$. $N_{G}$ means the norm with respect to $G$.

6) $f_{K \mid k}(G, \sigma)=\Pi_{\tau \in G} f_{K \mid k}(\tau, \sigma)$. 
is an exact sequence. Then we say that $E$ is a group extension of $A$ by $G$ and we denote $E(A, G \iota, \psi)$. By a homomorphism $\mu(\lambda, \nu)$ of $E(A, G, \iota, \psi)$ into $E^{\prime}\left(A^{\prime}, G^{\prime}, \iota^{\prime}, \psi^{\prime}\right)$ we mean a set of homomorphisms $\lambda: A \rightarrow A^{\prime}, \mu: E \rightarrow E^{\prime}, \nu: G \rightarrow G^{\prime}$ such that

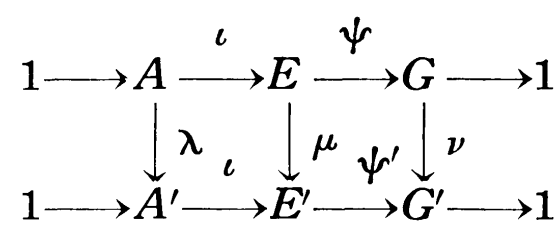

is a commutative diagram. In the rest of this section we assume that the group $A$ is abelian. Then $G$ can be considered as an operator group of $A$ by $a^{\sigma}=\iota^{-1}\left(u_{\sigma} \bullet \iota(a) \bullet u_{\sigma}^{-1}\right)(\sigma \in G, a \in A)$ where $\left\{u_{\sigma}\right\}$ is a system of representatives of $\left\{\boldsymbol{\psi}^{-1}(\sigma) \subset E ; \sigma \in G\right\}$.

$$
f(\sigma, \tau)=\iota^{-1}\left(u_{\sigma} u_{\tau} u_{\sigma \tau}^{-1}\right) \quad(\sigma, \tau \in G)
$$

is a 2-cocycle of $G$ over the $G$-group $A$. We call such a pair $\Sigma=\left(\left\{u_{\sigma}\right\},\{f(\sigma, \tau)\}\right)$ a frame of $E(A, G, \iota, \psi)$. Any two pairs $\left(\left\{u_{\sigma}\right\}\right.$, $\{f(\sigma, \tau)\})$ and $\left(\left\{v_{\sigma}\right\},\{g(\sigma, \tau)\}\right)$ are related by suitable $b_{\sigma}$ 's $\left(b_{\sigma} \in A\right)$ by $v_{\sigma}=\iota\left(b_{\sigma}\right) u_{\sigma}$, with $g(\sigma, \tau)=f(\sigma, \tau) b_{\sigma} b_{\tau}^{\sigma} b_{\sigma \tau}^{-1}$. For a fixed 2-cocycle $\{f(\sigma, \tau)\}$ there are as many different pairs $\left(\left\{u_{\sigma}\right\},\{f(\sigma, \tau)\}\right)$ as sets $\left\{b_{\sigma} ; \sigma \in G\left(b_{\sigma} \in A\right)\right\}$ which satisfies $b_{\sigma} b_{\tau}^{\sigma} b_{\sigma \tau}^{-1}=1$. The cohomology class of $\{f(\sigma, \tau)\}$ is uniquely determined by $E(A, G, \iota, \psi)$ and is independent of the choice of the representatives $\left\{\boldsymbol{u}_{\sigma}\right\}$. So we call it the 2-cohomology class associated with $E$.

Suppose that two group extensions $E(A, G, \iota, \psi)$ and $E^{\prime}\left(A^{\prime}, G^{\prime}, \iota^{\prime}\right.$, $\left.\psi^{\prime}\right)$, a homomorphism $\lambda: A \rightarrow A^{\prime}$ and an isomorphism $\nu: G \rightarrow G^{\prime}$ (into) are given such that $\lambda\left(a^{\sigma}\right)=\lambda(a)^{\nu(\sigma)}(a \in A, \sigma \in G)$ holds. Then there exists a homomorphism $\mu(\lambda, \nu): E(A, G, \iota, \psi) \rightarrow E^{\prime}\left(A^{\prime}, G^{\prime}, \iota^{\prime}, \psi^{\prime}\right)$ if and only if the with $E$ and $E^{\prime}$ associated 2-cocycles $\{f(\sigma, \tau)\}$ and $\left\{f^{\prime}\left(\sigma^{\prime}\right.\right.$, $\left.\left.\tau^{\prime}\right)\right\}$ satisfy $\lambda(f(\sigma, \tau)) \sim f^{\prime}(\nu(\sigma), \nu(\tau))(\sigma, \tau \in G)$. If we fix the frames $\Sigma^{\prime}=\left(\left\{\boldsymbol{u}_{\sigma}\right\},\{f(\sigma, \tau)\}\right)$ and $\Sigma^{\prime \prime}=\left(\left\{\boldsymbol{u}_{\sigma}^{\prime}\right\},\left\{f^{\prime}\left(\sigma^{\prime}, \boldsymbol{\tau}^{\prime}\right)\right\}\right)$ of $E$ and $E^{\prime}$ respectively such that $\lambda(f(\sigma, \tau))=f^{\prime}(\nu(\sigma), \gamma(\tau))$ holds, then a homomorphism $\mu$ is uniquely determined by $\mu: u_{\sigma} \rightarrow u_{\nu(\sigma)}^{\prime}$. It should be remarked that usually there are many such choices of $\mu$ 's according to that of $\Sigma$ 's.

Finally, for a given group $G$, an abelian $G$-group $A$ and a 2-cocycle $\{f(\sigma, \tau)\}$ of $G$ over $A$ there exists a group extension $E(A, G, \iota, \psi)$ such that 
(4) $\left\{\begin{aligned} E & =\cup_{\sigma} A^{*} \cdot u_{\sigma}\left(A^{*} \cong A\right), u_{\sigma} \cdot u_{\tau}=f(\sigma, \tau)^{*} u_{\sigma \tau}, u_{\sigma} \cdot a^{*}=\left(a^{\sigma}\right)^{*} \cdot u_{\sigma} \\ \iota(a) & =a^{*}, \psi\left(a^{*} u_{\sigma}\right)=\sigma .\end{aligned}\right.$

\section{§2. System of Weil groups}

1. Let $\{\Re, A(K)\}$ be a class formation. Let us assume that a group $W(K / k)$ is attached to each normal extension $K / k(k, K \in \Omega)$ with the following properties:

$\mathrm{A}_{1}$ There exist homomorphisms

$$
\begin{array}{ll}
\lambda_{K, k}: & A(K) \rightarrow W(K / k) \\
\pi_{K, k}: & W(K / k) \rightarrow G(K / k)
\end{array}
$$

such that

$$
1 \longrightarrow A(K) \stackrel{\lambda_{K, k}}{\longrightarrow} W(K / k) \stackrel{\pi_{K, k}}{\longrightarrow} G(K / k) \longrightarrow 1
$$

is exact. Moreover, let us take an arbitrary representative $u_{\sigma}$ in each $\pi_{K, k}^{-1}(\sigma)$ then

$$
u_{\sigma} \cdot(\lambda a) \cdot u_{\sigma}^{-1}=\lambda\left(a^{\sigma}\right) \quad \sigma \in G, a \in A(K)
$$

holds.

$\mathrm{A}_{2}$. Let

$$
\tau_{K, k}: \quad W(K / k) \rightarrow \lambda_{K, k} A(K)
$$

be the transfer mapping and let us put

$$
\mu_{K, k}=\boldsymbol{\varphi}_{K, k}^{-1} \circ \lambda_{K, k^{\circ}}^{-1} \boldsymbol{\tau}_{K, k}: \quad W(K / k) \rightarrow A(k) .
$$

Then

$$
1 \longrightarrow W(K / k)^{c} \stackrel{\iota}{\longrightarrow} W(K / k) \stackrel{\mu_{K, k}}{\longrightarrow} A(k) \longrightarrow 1
$$

is exact.

$\mathrm{B}_{1}$. In case II there exists an into-isomorphism

$$
\nu_{K, l, k}: \quad W(K / \ell) \rightarrow W(K / k)
$$

such that 
(9)

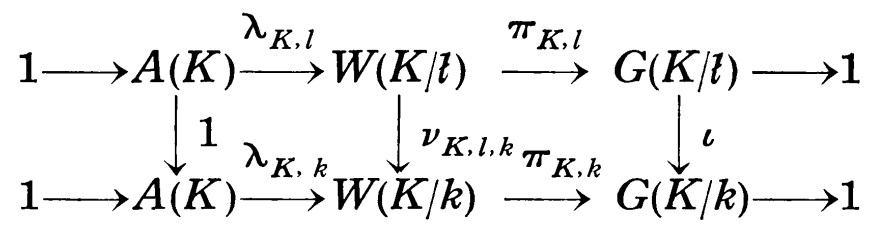

(exact)

is a commutative diagram. We remark that from (9) follows

$$
\nu_{K, l, k} W(K / \mathfrak{l})=\pi_{K, k}^{-1}(G(K / \mathfrak{l})) .
$$

$\mathrm{C}_{1}$. In case I there exists an onto-homomorphism

$$
\rho_{L, K, k}: W(L / k) \rightarrow W(K / k)
$$

such that

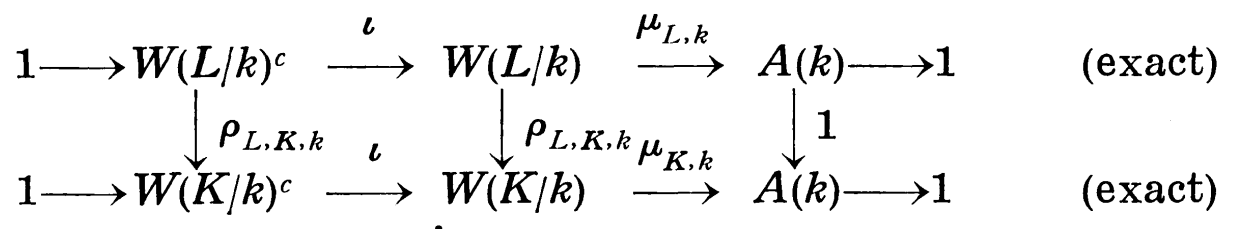

and

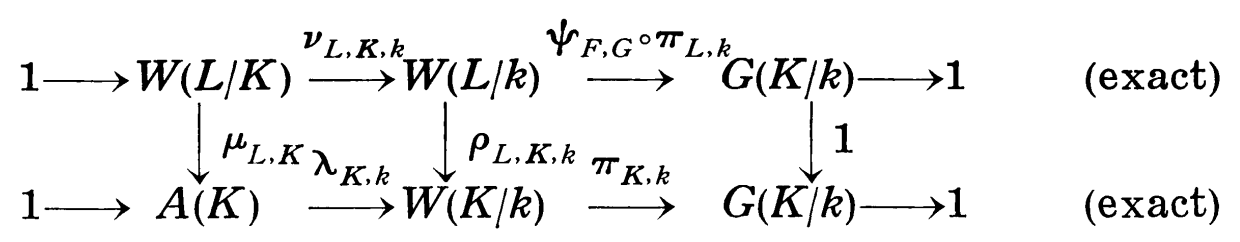

are commutative diagrams. We remark here that from (7) and (13) follows

$$
\text { Kernel } \rho_{L, K, k}=\text { Kernel } \mu_{L, K}=W(L / K)^{c} \text {. }
$$

$\mathrm{A}_{1}{ }^{\prime}$. Let the homomorphism

$$
\eta_{K}: \quad A(K) \rightarrow I^{\top}(K)
$$

be defined by the generalized norm-residue symbol $\eta_{K}(a)=(a, K)(a \in A(K))$. Let $I^{\prime}(K / k)=G\left(A_{K}^{\prime} k\right)$ for normal extension $K / k$. Then there exists a homomorphism

$$
\xi_{K, k}: \quad W(K / k) \rightarrow \Gamma(K / k)
$$

such that 


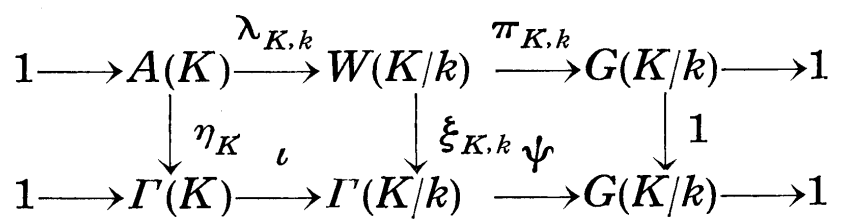

(exact)

(exact)

is a commutative diagram. then

$\mathrm{A}_{2}{ }^{\prime}$. Let $\Gamma(K / k)^{c}$ be the topological commutator group of $I^{\prime}(K / k)$

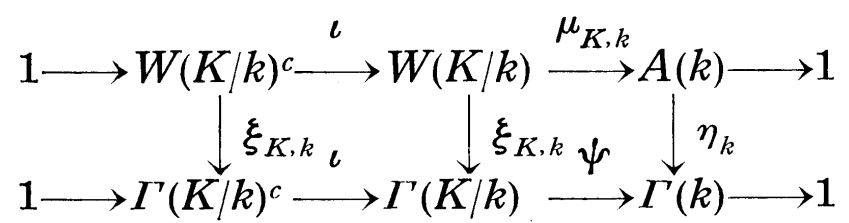

(exact)

(exact)

is a commutative diagram.

$\mathrm{B}^{\prime}$. In case II we can choose $\xi_{K, l}$ and $\xi_{K, k}$ such that

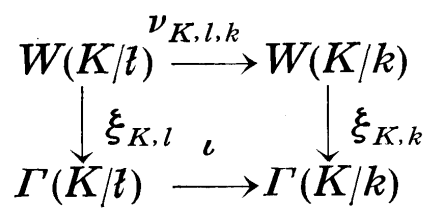

is a commutative diagram.

$\mathrm{C}^{\prime}$. In case I we can choose $\xi_{L, k}$ and $\xi_{K, k}$ such that

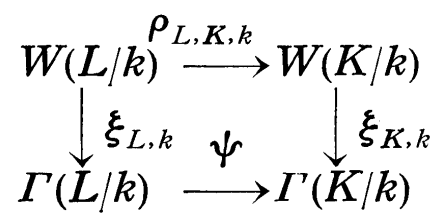

is a commutative diagram.

If all these properties are satisfied we call the system $\{W(K / k)\}$ a system of Weil groups associated with the class formation (cf. Weil [12])

2. Now we can prove the following existence theorem:

THEOREM 1. For any given class formation $\{\mathfrak{\Omega}, A(K)\}$ there exists always a system of Weil groups $\{W(K / k)\}$ associated with it.

(PROOF) (i) Let $\left\{f_{K / k}\right\}$ be a system of fundamental 2-cocycles and $(a, k)$ be the generalized norm-residue symbol defined by them. Since $f_{K \mid k}(\sigma, \tau)$ is a 2-cocycle of $G(K / k)$ over $A(K)$ we can define a 
group extension $W(K / k)$ of $A(K)$ by $G(K / k)$ defined by $f_{K / k}(\sigma, \tau)$ :

$$
\left\{\begin{array}{l}
W(K / k)=\bigcup_{\sigma \in G} A(K)^{*} \cdot u_{\sigma} \\
u_{\sigma} \cdot u_{\tau}=f_{K / k}(\sigma, \tau)^{*} \cdot u_{\sigma \tau}, u_{\sigma} \cdot a^{*}=\left(a^{\sigma}\right)^{*} \cdot u_{\sigma} \\
\lambda_{K, k} a=a^{*}, \quad \pi_{K, k}\left(a^{*} \cdot u_{\sigma}\right)=\sigma
\end{array}\right.
$$

where $A(K)$ * is an isomorphic replica of $A(K)$ as abelian $G$-group. Thus we can construct $W(K / k)$ satisfying (3). In the following we fix a frame $\Sigma_{K / k}=\left(\left\{u_{\sigma}\right\},\left\{f_{K / k}(\sigma, \tau)\right\}\right)$. Clearly $\lambda_{K, k}$ and $\pi_{K, k}$ are independent of the choice of a frame.

(ii) The transfer mapping $\tau_{K, k}: W(K / k) \rightarrow A(K)^{*}=\lambda_{K, k} A(K)$ is defined by

$$
\tau_{K, k}\left(a^{*}\right)=\left(N_{G} a\right)^{*}, \tau_{K, k}\left(u_{\sigma}\right)=\prod_{\tau \in G} u_{\tau} u_{\sigma} u_{\tau \sigma}^{-1}=f_{K \mid k}(G, \sigma)^{*}
$$

By the isomorphism theorem we have $A(K)^{G}=\prod_{\sigma \in G} f(G, \sigma) \bullet\left(N_{G} A(K)\right)$ which implies $\tau_{K, k} W(K / k)=\lambda_{K, k} A(K)^{G}$. Next consider the kernel of $\tau_{K, k^{*}}$ So let $\tau_{K, k}\left(a^{*} \cdot u_{\sigma}\right)=\tau\left(a^{*}\right) \cdot \tau\left(u_{\sigma}\right)=1$. It follows from the same theorem that $\sigma \in G^{c}$. Let $\sigma=\prod_{j} \tau_{j}\left(\sigma_{j} \rho_{j} \sigma_{j}^{-1} \rho_{j}^{-1}\right)^{\varepsilon_{j}} \tau_{j}^{-1}$, then we have $u_{\sigma}$ $=a_{1}^{*} \cdot c, c=\prod_{j} u_{\tau_{j}}\left(u_{\sigma_{j}} u_{\rho_{j}} u_{\sigma_{j}}^{-1} u_{\rho_{j}}^{-1}\right)^{\varepsilon_{j}} u_{\tau_{j}}^{-1}$ and $a_{1} \in A$. Hence it follows that $a^{*} \cdot u_{\sigma}=a_{2}^{*} \cdot c$ where $c \in W(K / k)^{c}$ and $a_{2} \in A(K)$ with $\tau_{K, k} a_{2}^{*}=1$. To prove Kernel $\tau=W(K / k)^{c}$ it is enough to see that $\left(N_{G} a\right)^{*}=1$ implies $a^{*} \in W(K / k)^{c}$. Since $H^{-1}(G, A(K)) \cong A(K)^{N_{G^{-1}}} / I(A(K))\left(I(A)=\prod_{\sigma \mathrm{e} G} A^{\sigma-1}\right)$ is isomorphic to $H^{-3}(G, Z)=H_{2}(G, Z)$ by the Theorem of Tate, we have

$$
A(K)^{N_{G^{-1}}}=\left\{g^{-3} \cup f_{K / k}\right\} \cdot I(A(K)) \text {. }
$$

Here $g^{-3}$ is a 2-cocycle of $G$ over $Z$, i. e. $g^{-3}=\sum_{\sigma, \tau} a_{\sigma, \tau}(\sigma, \tau)\left(a_{\sigma, \tau} \in Z\right)$ with $\delta g^{-3}=\sum_{\sigma, \tau} a_{\sigma, \tau}\{(\tau)-(\sigma \tau)+(\sigma)\}=0$. By the explicit formula of cup-product (see e.g. Artin-Tate [3]) we have

$$
\left(\sum a_{\sigma, \tau}(\sigma, \tau)\right) \cup f=\prod_{\sigma, \tau}\left\{f(\sigma, \tau)^{\sigma^{-1} \tau^{-1}}\right\}-a_{\sigma, \tau}=\prod_{\sigma, \tau}\left(u_{\sigma \tau}^{-1} u_{\tau} u_{\sigma}\right)^{-a_{\sigma, \tau}} .
$$

This term belongs to $W(K / k)^{c}$ since it becomes the unity element by taking $\bmod W(K / k)^{c}$ by means of $\delta g^{-3}=0$. Also $\left(a^{*}\right)^{\sigma-1}=u_{\sigma} a^{*} u_{\sigma}^{-1} a^{-1} \in$ 
$W(K / k)^{c}$ implies $I(A(K)) \subset W(K / k)^{c}$. Hence we have proved Kernel $\tau$ $=W(K / k)^{c}$. Since Kernel $\mu_{K, k}=$ Kernel $\tau_{K, k}=W(K / k)^{c}$ we get the exactness of (7) (cf. Kawada [6], p. 92). Evidently the homomorphism $\mu_{K, k}$ is independent of the choice of a frame $\Sigma_{K / k}$ of $W(K / k)$.

(iii) $\mathrm{B}_{1}$. Firstly, let us normalize $f_{K \mid l}=\operatorname{res}_{H, G} f_{K \mid k}$ and let us choose $\Sigma_{K \mid k}=\left(\left\{u_{\sigma}\right\},\left\{f_{K \mid k}(\sigma, \tau)\right\}\right)$ and $\Sigma_{K / l}=\left(\left\{v_{\rho}\right\},\left\{f_{K / l}(\sigma, \tau)\right\}\right)$ of $W(K / k)$ and $W(K / \ell)$ respestively:

$$
W(K / k)=\underset{\sigma \mathrm{e} G}{\bigcup} A(K)^{*} \cdot u_{\sigma}, W(K / t)=\bigcup_{\rho \text { e } H} A(K)^{*} u_{\rho}^{*} .
$$

Then we can define an into-isomorphism $\nu_{K, l, k}(1, \iota)$ of $W(K / t)(A(K), H)$ into $W(K / k)(A(K), G)$ by

$$
\nu\left(a^{+}\right)=a^{*}(a \in A(K)), \quad \nu\left(u_{\rho}^{+}\right)=u_{\rho}(\rho \in H) .
$$

It is then easy to verify (9). It should be remarked that $\nu_{K, l, k}$ depends on the choice of the frames $\Sigma_{K / k}$ and $\Sigma_{K / l}$.

(iv) $\mathrm{C}_{1}$. Let us normalize $f_{L / K}=\operatorname{res}_{H, G} f_{L / k}$ and let us choose $\Sigma_{L / k}=\left(\left\{u_{\sigma}\right\},\left\{f_{L / k}\right\}\right)$ and $\Sigma_{L / K}=\left(\left\{u_{\rho}^{\mid l}\right\},\left\{f_{L / K}\right\}\right)$ of $W(L / k)$ and $W(L / K)$ respectively :

$$
W(L / k)=\bigcup_{\sigma \mathrm{e} G} A(L)^{*} \cdot u_{\sigma}, W(L / K)=\bigcup_{\rho \mathrm{e} H} A(L)^{\cdots} \cdot u_{\rho}^{*} \cdot
$$

Let us fix for a moment a system of representatives $\{\bar{\sigma}\}$ of cosets: $G=\bigcup H \bar{\sigma}$ and put

$$
\left\{\begin{array}{l}
g(\sigma, \tau)=f_{L / k}(\sigma, \tau)^{m_{\bullet}}(\delta h)(\sigma, \tau) \quad(m=[L: K]) \\
h(\sigma)=f_{L / k}(H, \bar{\sigma}) \cdot f_{L / k}(\sigma, H)^{-1}
\end{array}\right.
$$

Then it follows that $g(\sigma, \tau)=g(\bar{\sigma}, \bar{\tau})$ and so $g(\sigma, \tau)=\operatorname{infl}_{G, F} \hat{g}(\widetilde{\sigma}, \widetilde{\tau})$ where $\widetilde{\sigma}$ denotes the class of $\sigma \bmod H$. Hence we can normalize

$$
f_{K / k}(\widetilde{\sigma}, \widetilde{\tau})=g(\bar{\sigma}, \bar{\tau}) \text {. }
$$

By a known formula (see e.g. Hochschild-Nakayama [5], Lemma 7) we have

$$
\operatorname{infl}_{G, F^{\circ}} \boldsymbol{\varphi}_{L, K}\left(f_{K / k}(\tilde{\sigma}, \widetilde{\tau})\right)=\lambda_{L, K^{\circ}}^{-1} \boldsymbol{\tau}_{L, K^{\circ}} \nu_{L, K, k}^{-1}\left(u_{\bar{\sigma}} u_{\bar{\tau}} u_{\bar{\sigma}}^{-\frac{1}{\tau}}\right) .
$$

We choose a frame $\Sigma_{K / k}=\left(\left\{v_{\tilde{\sigma}}\right\},\left\{f_{K / k}\right\}\right)$ of $W(K / k)$ :

$$
W(K \mid k)=\underset{\tilde{\sigma} \in F}{\bigcup} A(K)^{*} \cdot v_{\tilde{\sigma}} \text {. }
$$


By $\mathrm{B}_{1}$ we can decompose $W(L / k)=\bigcup_{\bar{\sigma}} \nu_{L, K, k}(W(L / K)) \cdot u_{\bar{\sigma}}$. As in the considerations in $\S 1,2$ we can define a homomorphism of $W(L / k)$ into $W .(K / k)$ as an extension of

$$
\rho^{*}=\mu_{L, K^{\circ} \nu_{L, K, k}^{-1}:} \nu_{L, K, k}(W(L / K)) \rightarrow A(K)
$$

by means of

$$
\rho\left(u_{\bar{\sigma}}\right)=v_{\tilde{v}} \quad(\sigma \in G),
$$

because using (27) we can verify the corresponding conditions:

$$
\rho^{*}\left(u_{\bar{\sigma}} \cdot u_{\bar{\tau}} \cdot u_{\bar{\sigma}}^{-\frac{1}{\sigma}}\right)=f_{K \mid k}(\widetilde{\sigma}, \widetilde{\tau})^{*}, \rho^{*}\left(u_{\bar{\sigma}} \cdot\left(a^{*} \cdot u_{\rho}\right) \cdot u_{\bar{\sigma}}{ }^{1}\right)=v_{\tilde{\sigma}} \cdot \rho^{*}\left(a^{*} \cdot u_{\rho}\right) \cdot v_{\bar{\sigma}}^{-1}
$$

for $\sigma, \tau \in G$. Then from the definition of $\rho=\rho_{L, K, k}$ follows the commutativity of (13).

Next we shall verify (2). By a known formula of Akizuki-Witt [1] $g(\sigma, \tau)$ in [25) can be expressed in another form:

$$
g(\sigma, \tau)=f_{L / k}(H, \bar{\sigma}) \cdot f_{L / k}(H, \overline{\sigma \tau})^{-1} \cdot f_{L / k}(H \bar{\sigma}, \bar{\tau}) .
$$

Hence we have

$$
f_{L \mid k}(G, \bar{\sigma})=\hat{g}(F, \widetilde{\sigma})=f_{K / k}(F, \widetilde{\sigma})
$$

by the normalization (26). Now compare the two mappings $\mu_{L, k}$ and $\mu_{K, k} \circ \rho_{L, K, k^{\circ}}$ On the subgroup $\nu_{L, K, k}(W(L / K))$ we have by (28) $\mu_{K, k^{\circ}} \rho_{L, K, k}=\mu_{K, k^{\circ}} \mu_{L, K^{\circ}} \nu_{L, K, j}^{-1}=\mu_{L, k}$ using the transitivity of the transfer mappings. On the set $\left\{\boldsymbol{u}_{\bar{\sigma}}\right\}$ we have by (21) and (29) $\mu_{K, k} \rho_{L, K, k}$ $\left(u_{\bar{\sigma}}\right)=\mu_{K, k}\left(v_{\tilde{\sigma}}\right)=f_{K \mid k}(F, \widetilde{\sigma})$ and $\mu_{L, k}\left(u_{\bar{\sigma}}\right)=f_{L / k}(G, \bar{\sigma})$ which are the same by (30). Thus we have proved (12).

We should remark here that the definition of $\rho_{L, K, k}$ depends on the choice of the frames $\Sigma_{L / k}^{\prime}, \Sigma_{L / K}$ and $\Sigma_{K / k}$ but does not depend on the choice of representatives $\{\bar{\sigma}\}$ of cosets $G \bmod H$.

(v) To show the existence of $\xi_{K, k}$ we need a generalized theorem of Šavarevič (see, e.g. Hochschild-Nakayama [5], Theorem 3.2 or Artin-Tate [3]). Namely let $f_{K, k}(\widetilde{\sigma}, \widetilde{\tau})(\widetilde{\sigma}, \widetilde{\tau} \in G)$ be a fundamental 2cocycle of $G$ over $A(K)$, then

$$
\mathrm{f}_{K / k}(\widetilde{\sigma}, \widetilde{\tau})=\left(f_{K \mid k}(\widetilde{\sigma}, \widetilde{\tau}), K\right) \in \Gamma(K)
$$

is a 2-cocycle of $G$ over $\Gamma(K)$ associated with the group extension $\Gamma(K / k)(\Gamma(K), G, \iota, \psi)$. Since we need the proof itself we repeat the known proof. By a known formula of Akizuki-Witt [1] $g(\sigma, \tau)$ in 
(25) can be expressed in another form

$$
g(\sigma, \tau)=f_{L, k}(H, \gamma) \cdot\left(f_{L, k}(\bar{\sigma}, \bar{\tau}) / f_{L / k}(\gamma, \overline{\sigma \tau})\right)^{H}
$$

where $\gamma=\bar{\sigma} \cdot \bar{\tau} \cdot \overline{\sigma \tau}^{-1} \in H$. Now take $k \subset K \subset L \subset A_{K}$ and denote here $G=G(K / k)$. Then from (32) follows $(g(\widetilde{\sigma}, \widetilde{\tau}), L / K)=\bar{\sigma} \cdot \bar{\tau} \cdot \overline{\sigma \tau}{ }^{-1} \in H$.

Since $f_{K \mid k}(\tilde{\sigma}, \tilde{\tau})=g(\tilde{\sigma}, \widetilde{\tau}) \cdot c_{\tilde{\sigma}} \cdot c_{\tilde{\tau}} \bullet c_{\tilde{\sigma} \tilde{\tau}}^{-1}\left(c_{\tilde{\sigma}} \in A(K)\right)$ we have

$$
\left(f_{K / k}(\tilde{\sigma}, \widetilde{\tau}), L / K\right)=\left(\rho_{\tilde{\sigma}} \cdot \bar{\sigma}\right) \cdot\left(\rho_{\tilde{\tau}} \cdot \bar{\tau}\right) \cdot\left(\rho_{\tilde{\sigma} \tau} \cdot \overline{\sigma \tau}\right)^{-1} \in H
$$

where we put $\rho_{\tilde{\sigma}}=\left(c_{\tilde{\sigma}}, L / K\right) \in H$. Take the limit with respect to all $\left\{L ; K \subset L \subset A_{K}\right\}$ we have

$$
\left(f_{K / k}(\widetilde{\sigma}, \tilde{\tau}), K\right)=\sigma^{*} \cdot \tau^{*} \cdot(\sigma \tau)^{*-1} \in \Gamma(K)
$$

where $\sigma^{*} \in \boldsymbol{\Gamma}(K / k)$ means some element with $\psi\left(\sigma^{*}\right)=\tilde{\sigma} \in G(K / k)$. This proves our proposition.

Let us put in (34) $U_{\widetilde{\sigma}}=\sigma^{*}(\widetilde{\sigma} \in G)$, then

$$
\Gamma(K / k)=\bigcup_{\tilde{\sigma} \mathrm{e} G} \Gamma(K) \cdot U_{\tilde{\sigma}}, U_{\tilde{\sigma}} \cdot U_{\tilde{\tau}}=f_{K / k}(\widetilde{\sigma}, \tilde{\tau}) \cdot U_{\tilde{\sigma} \tilde{\tau}} \cdot
$$

By the general method shown in $\S 1,2$ we can define the homomorphism $\xi_{K, k}: W(K / k) \rightarrow \Gamma(K / k)$ as an extension of $\eta_{K}$ by $\xi_{K, k}\left(u_{\tilde{\sigma}}\right) \rightarrow U_{\tilde{\sigma}}$ $(\tilde{\sigma} \in G)$. Then the commutativity of (16) holds.

We should remark here that $\xi_{K, k}$ depends on the choice of frames $\Sigma_{K / k}^{\prime}=\left(\left\{u_{\tilde{\sigma}}\right\},\left\{f_{K / k}\right\}\right)$ of $W(K / k)$ and $\widetilde{\Sigma}_{K / k}=\left(\left\{U_{\tilde{\sigma}}\right\},\left\{f_{K / k}\right\}\right)$ of $\Gamma(K / k)$ where $f_{K / k}$ is defined by (31). If we fix $\Sigma_{K / k}$ there are as many possibilities of $\xi_{K, k}$ as different frames $\left(\left\{B_{\tilde{\sigma}} U_{\tilde{\sigma}}\right\},\left\{f_{K / k}\right\}\right)$ of $\Gamma(K / k)$ where $B_{\tilde{\sigma}} \in \Gamma(K)$ and

$$
B_{\tilde{\sigma}} B_{\tilde{\tau}}^{\tilde{\sigma}} B_{\tilde{\sigma} \tau}^{-1}=1 \quad(\widetilde{\sigma}, \tilde{\tau} \in G) .
$$

(vi) LEMMA 2. If a homomorphism $\xi_{K, k}: W(K / k) \rightarrow \Gamma(K / k)$ satisfies $\mathrm{A}_{1}{ }^{\prime}$ then the property $\mathrm{A}_{2}{ }^{\prime}$ is satisfied automatically for this $\xi_{K, k}$.

(PROOF) To prove the commutativity of (17) it is enough to see

$$
\begin{array}{cc}
\psi(a, K)=\left(N_{G} a, k\right) \quad(a \in A(K)) \\
\psi U_{\tilde{\sigma}}=\left(f_{K / k}(G(K / k), \tilde{\sigma}), k\right) & (\tilde{\sigma} \in G(K / k))
\end{array}
$$

where $\psi=\psi\left(\Gamma^{\prime}(k), \Gamma(K / k)\right)^{7}$. Here (37) is a known property of the norm-residue symbol. To prove (38) let $k \subset K \subset L \subset A_{K}, L / k$ be normal and put $L^{\prime}=A_{k} \cap L$. Then (38) is equivalent to

7) $\psi(F, G)$ means $\psi_{F, G}$, i. e. the cannonical homomorphism of $G$ on $F$. 


$$
\psi^{\prime} U_{\tilde{\sigma}}=\left(f_{K^{\prime} / k}(G(K / k), \tilde{\sigma}), L^{\prime} / k\right)
$$

for all such $L$ where $\psi^{\prime}=\psi\left(G\left(L^{\prime} / k\right), I^{\prime}(K / k)\right)$. From the choice of $U_{\tilde{\sigma}}=\sigma^{*}$ in (v) follows $\psi^{\prime} U_{\tilde{\sigma}}=\psi^{\prime \prime}\left(\rho_{\tilde{\sigma}} \cdot \bar{\sigma}\right)$ in (33). Put $F=G(K / k)$. Then from $f_{K ! k}=g \cdot \delta c$ it follows that $f_{K / k}(F, \tilde{\sigma})=g(F, \tilde{\sigma}) \cdot N_{F} c_{\tilde{\sigma}}$. On the other hand we have $\psi^{\prime \prime}\left(\rho_{\tilde{u}}\right)=\psi^{\prime \prime}\left(c_{\tilde{\imath}}, L / K\right)=\left(N_{F} c_{\tilde{u}}, L^{\prime} \mid k\right)$ and $\psi^{\prime \prime}(\bar{\sigma})=\left(f_{L / k}(G, \bar{\sigma})\right.$, $\left.L^{\prime} \mid k\right)$ by definition. Hence using these relations and (30) we have

$$
\psi^{\prime \prime}\left(\rho_{\tilde{\nu}} \cdot \bar{\sigma}\right)=\left(g(F, \tilde{\sigma}) N_{F} c_{\tilde{\nu}}, L^{\prime} / k\right)=\left(f_{K / k}(F, \tilde{\sigma}), L^{\prime} / k\right), \quad \text { q. e. d. }
$$

(vii) $\mathrm{B}^{\prime}$. Let us fix frames $\Sigma_{K / k}$ and $\Sigma_{K / z}$ as in (iii) for the normalization $f_{K / \xi}=\operatorname{res}_{H, C} f_{K / k}$, and choose the frames $\widetilde{\Sigma}_{K / k}$ of $\Gamma(K / k)$ and $\hat{\Sigma}_{K / \mathfrak{t}}$ of $(K / \mathfrak{l})$ as in (v). If we define $\xi_{K, k}, \xi_{K, t}$ and $\nu_{K, k, k}$ by

$$
\nu_{K \downarrow, k}\left(u_{\rho}^{\dagger}\right)=u_{\rho}, \xi_{K z}\left(u_{\rho}^{\dagger}\right)=U_{\rho}^{\vdash}, \xi_{K, k}\left(u_{\sigma}\right)=U_{\sigma}
$$

then we have $\iota\left(U_{\rho}^{*}\right)=U_{\sigma}$ and $\mathrm{B}^{\prime}$ holds.

$\mathrm{C}^{\prime}$. Let us fix the frames $\Sigma_{L / k}^{\prime}, \Sigma_{L / K}$ and $\Sigma_{K / k}^{\prime}$ as in (iv) for $f_{L / K}$ $=\operatorname{res}_{H, G} f_{L / k}$ and for $f_{K / k}$ as in (26). Let us choose the frames $\widetilde{\Sigma}_{L / k}$ $=\left(\left\{U_{\sigma}\right\},\left\{f_{L / k}\right\}\right)$ and $\bar{\Sigma}_{K / k}=\left(\left\{V_{\tilde{\sigma}}\right\},\left\{f_{K / k}\right\}\right)$ of $\Gamma(L / k)$ and $I(K / k)$ respectively such that

$$
\psi\left(U_{\bar{\sigma}}\right)=V_{\tilde{\sigma}} \quad(\sigma \in G, \psi=\psi(\Gamma(K / k), \Gamma(L / k))
$$

holds. Then we can verify the commutativity of (19) immediately. That we can choose $\widehat{\Sigma}_{K / k}$ and $\tilde{\Sigma}_{L / k}^{\prime}$ satisfying (41) can be shown as follows. Let us take $\bar{\Sigma}_{L / k}$ as in (v) from $\Sigma_{L / k}$ of $W(L / k)$ and define $\xi_{L, k}$ as there. Next put $V_{\tilde{\sigma}}=\psi\left(U_{\bar{\sigma}}\right)$. Then from

$$
\psi\left(U_{\bar{\sigma}} U_{\bar{\tau}} U_{\overline{\sigma \tau}}^{-1}\right)=\left(\mu_{L, K}\left(u_{\bar{\sigma}} u_{\bar{\tau}} u_{\bar{\sigma} \tau}^{-1}\right), K\right)=\left(f_{K / k}(\tilde{\sigma}, \tilde{\tau}), K\right)
$$

follows that

$$
V_{\bar{\sigma}} \cdot V_{\tilde{\tau}}=\psi\left(U_{\bar{\sigma}} \cdot U_{\bar{\tau}}\right)=\psi\left(U_{\bar{\sigma}} \cdot U_{\overline{\bar{\tau}}} \cdot U_{\overline{\sigma \tau}}^{-1} \cdot U_{\bar{\sigma} \tilde{\tau}}\right)=\left(f_{K / k}(\widetilde{\sigma}, \widetilde{\tau}), K\right) \cdot V_{\tilde{\sigma} \tilde{\tau}} \cdot
$$

This shows that $\left(\left\{V_{\tilde{\sigma}}\right\},\left\{f_{K / k}\right\}\right)$ gives a desired frame of $\Gamma(K / k)$. q.e.d.

Let $\{W(K / k)\}$ be a system of Weil groups. If we can choose the homomorphisms $\nu, \rho$ once and for all such that the conditions

$\mathrm{B}_{2}$. Let $k \subset l \subset j \subset K$ and $K / k$ be normal. Then

$$
\nu_{K, l, k^{\circ}} \nu_{K, j, l}=\nu_{K, j, k}
$$

holds.

$\mathrm{C}_{2}$. Let $k \subset K \subset L \subset M$ and $M / k, L / k, K / k$ are all normal. Then 


$$
\rho_{L, K, k} \circ \rho_{M, L, k}=\rho_{M, K, k} .
$$

$\mathrm{C}_{3}$. Let $k \subset l \subset K \subset L$ and $L / k, K / k$ be normal. Then

$$
\rho_{L, K, k^{\circ}} \nu_{L, l, k}=\nu_{K, l, k} \circ \rho_{L, K, l}
$$

holds. (In case $K=l \quad \mathrm{C}_{3}$ is contained in $\mathrm{C}_{1}$ ). are satisfied then we call $\{W(K / k)\}$ a strong system of Weil groups.

REMARK. In a strong system of Weil groups we can choose the homomorphisms $\xi_{K, k}$ once and for all such that $\mathrm{B}^{\prime}$ and $\mathrm{C}^{\prime}$ hold.

For, what we have proved is that if we fix the frames $\Sigma_{K / k}$ of $W(K / k)$ for all $K / k$ then we can choose (i) $\widetilde{\Sigma}_{K / k}$ and $\widetilde{\Sigma}_{K / t}$ of $\Gamma(K / k)$ and $\Gamma(L / t)$ respectively so as to satisfy $\mathrm{B}^{\prime}$ and (ii) $\tilde{\Sigma}_{L / k}$ and $\tilde{\Sigma}_{K / k}$ of $\Gamma(L / k)$ and $\Gamma(K / k)$ so as to satify $\mathrm{C}^{\prime}$. What we want to prove is that for each individual $\{k, t, K\}$ or $\{k, K, L\}$ we can choose the frame $\widetilde{\Sigma}_{K / k}$ for each $K / k$ once for all such that $\mathrm{B}^{\prime}$ and $\mathrm{C}^{\prime}$ holds for these frames.

For that purpose let us consider the set $\Xi_{K, k}$ of all homomorphisms $\xi_{K, k}$ satisfying $\mathrm{A}^{\prime}$. If we fix a frame $\Sigma_{K / k}$ of $W(K / k), \xi_{K, k}$ depends on the choice among different frames $\widetilde{\Sigma}_{K / k}$ in $\Gamma(K / k)$ with a fixed 2-cocycle $f_{K / k}$ as in (31) and this depends on 1-cocycle $\left\{B_{\tilde{\sigma}}\right\}$ of $G$ in $\Gamma^{\top}(K)$. Since $\Gamma^{\top}(K)$ is compact we can introduce a natural topology in $\Xi_{K, k}$ such that $\Xi_{K, k}$ is compact. Let $\mathfrak{Q}=\left\{K_{\lambda}\right\}$ be the set of all finite normal extesnions $K_{\lambda} / k_{0}$ in $\Re$ and put $\Xi_{\lambda}=\Xi_{K_{\lambda}, k_{0}}$. If $K_{\lambda} \subset K_{\mu}$ $(\in \mathfrak{Q})$ then we can define the continuous mapping $\psi_{\lambda, \mu}$ of $\Xi_{\mu}$ into $\Xi_{\lambda}$ by (41). It is easy to see that $\left\{\boldsymbol{\Xi}_{\lambda}, \psi_{\lambda, \mu}\right\}$ makes an inverse mapping system. Since each $\Xi_{\lambda}$ is compact the limit space is not empty. Take an element in its limit space. Then we have a set $\left\{\xi_{K_{\lambda}, k_{0}}\right.$; $\left.K_{\lambda} \in \mathbb{R}\right\}$ such that (41) holds for each pair $\left\{\xi_{K_{\mu}, k_{0}}, \xi_{K_{\lambda}, k_{0}} ; K_{\lambda} \subset K_{\mu}\right\}$. Thus we have chosen $\xi_{K_{\lambda}, k_{0}}$. For a general normal extension $K / k$ we can take $k_{0} \subset k \subset K \subset L(L \in \mathfrak{R})$ and put

$$
\widetilde{\Sigma}_{K, k}=\psi_{L, K^{\circ}} \text { res } \widetilde{\Sigma}_{L, k 0}
$$

where $\psi_{L, K}$ means the mapping $\Xi_{L, k} \rightarrow \Xi_{K, k}$ defined by (41) and res means the restriction from $\Gamma\left(L / k_{0}\right)$ on $\Gamma(L / k)$. We can then prove in the usual way that the definition (45) does not depend on the choice of $L \in \mathfrak{Q}$ and thus defined family $\left\{\xi_{K, k}\right\}$ satisfies $\mathrm{B}^{\prime}, \mathrm{C}^{\prime}$, q. e. d. 
3. Let $\{\Omega, A(K)\}$ be a class formation and $\eta_{K}(a)=(a, K)$ be a generalized norm-residue symbol defined there. Suppose that two systems of Weil groups $\{W(K / k)\}$ and $\left\{W^{\prime}(K / k)\right\}$ associated with this class formation be given. Let us denote the groups and homomorphisms in $A, B, C, A^{\prime}, B^{\prime}, C^{\prime}$ with respect to $W^{\prime}(K / k)$ with prime.

We call $\{W(K / k)\}$ and $\left\{W^{\prime}(K / k)\right\}$ are isomorphic systems if there exists an isomorphism

$$
\Phi_{K, k}: W(K / k) \rightarrow W^{\prime}(K / k) \quad \text { (onto) }
$$

for each $K / k$ such that the following conditions $\mathrm{A}^{*}, \mathrm{~B}^{*}, \mathrm{C}^{*}, \mathrm{~A}^{\prime *}$ hold:

$\mathrm{A}^{*}: \quad \Phi_{K, k}^{\circ} \lambda_{K, k}=\lambda_{K, k}^{\prime}, \pi_{K, k}=\pi_{K, k}^{\prime} \circ \Phi_{K, k}, \mu_{K, k}=\mu_{K, k^{\circ}}^{\prime} \Phi_{K, k}$

$\mathrm{B}^{*}: \quad \Phi_{K, k^{\circ}} \nu_{K, z, k}=\nu_{K, k_{k}}^{\prime} \Phi_{K z}$

$\mathrm{C}^{*}: \quad \Phi_{K, k^{\circ}} \rho_{L, K, k}=\rho_{L, K, k}^{\prime}{ }^{\circ} \Phi_{L, K}$

$\mathrm{A}^{\prime *}: \quad \xi_{K, k}=\xi_{K, k}^{\prime} \Phi_{K, k} \cdot\left(\eta_{K}=\eta_{K}^{\prime}\right)$

THEOREM 2. Let us assume that the axioms $\mathrm{P}$ and $\mathrm{P}^{\prime}$ are satisfied in. . Then any two strong systems of Weil groups $\{W(K / k)\}$ and $\left\{W^{\prime}(K / k)\right\}$ are isomorphic.

(PROOF) (i) We may assume that the system $\{W(K / k)\}$ is the one defined in the proof of Theorem 1. Let us choose an arbitrary frame $\Sigma_{K, k}^{\prime}=\left(\left\{u_{\sigma}^{\prime}\right\},\left\{g_{K / k}(\sigma, \tau)\right\}\right)$ in each $W^{\prime}(K / k)$ :

$$
W^{\prime}(K / k)=\bigcup_{\sigma \in G} A(K)^{\prime} \cdot u_{\sigma}^{\prime}, \quad u_{\sigma}^{\prime} \cdot u_{\tau}^{\prime}=g_{K / k}(\sigma, \tau)^{\prime} \cdot u_{\sigma \tau}^{\prime} \cdot
$$

From the conditions $\mathrm{B}_{1}, \mathrm{C}_{1}$ follows that $\nu_{K, z, k}^{\prime}$ induces $g_{K \mid z} \sim \operatorname{res}_{H, G} g_{K \mid k}$ and $\rho_{L, K, k}^{\prime}$ induces $\operatorname{infl}_{G, F} g_{L / k} \sim g_{K / k}^{m}(m=[L: K])$. Moreover, $g_{K / k}$ is a generic 2-cocycle in $H^{2}(G, A(K))$. For, if it were not so $g_{K / z} \sim 1$ would hold on some cyclic subgroup $H=G(K / \mathfrak{l})$ (by the above relation). On the other hand we have $A(K)^{H}=\bigcup_{\rho \in H} g_{K / z}(H, \rho) \cdot N_{H} A(K)$ by $\mathrm{A}_{2}$. Since $g_{K / z} \sim 1$ we would have $g_{K / z}(H, \rho) \in N_{H} A(K)$ which would imply $A(K)^{H}=N_{H} A(K)$. But this contradicts with the isomorphism theorem $A(K)^{H} / N_{H} A(K) \cong H$.

We shall prove next that $g_{K / k} \sim f_{K / k}$ holds for every $K / k$. Let us first assume that $G=G(K / k)$ is cyclic and so put $G=\left\{1, \sigma, \sigma^{2}, \cdots\right.$, $\left.\sigma^{n-1}\right\}$. Then we can normalize 


$$
\begin{aligned}
& f_{K / k}\left(\sigma^{i}, \sigma^{j}\right)=1(i+j<n) ;=\lambda_{K, k}(a)(i+j \geqq n)(a \in A(k)) \\
& g_{K / k}\left(\sigma^{i}, \sigma^{j}\right)=1(i+j<n) ;=\lambda_{K, k}(b)(i+j \geqq n)(b \in \mathrm{A}(k)) .
\end{aligned}
$$

By $\mathrm{A}_{2}^{\prime}$ we have

$$
\begin{aligned}
& \psi \circ \xi_{K, k}\left(u_{\sigma}\right)=\left(f_{K / k}(G, \sigma), k\right)=(a, k) \\
& \psi \circ \xi_{K, k}^{\prime}\left(u_{\sigma}^{\prime}\right)=\left(g_{K / k}(G, \sigma), k\right)=(b, k)
\end{aligned}
$$

where $\psi=\psi\left(\Gamma^{\prime}(k), \Gamma(K / k)\right.$. From the commutativity of (16) follows

$$
\psi \circ \xi_{K, k}\left(u_{\sigma}\right) \equiv \pi_{K, k}\left(u_{\sigma}\right) \equiv \sigma \quad\left(\bmod G\left(A_{k} / K\right)\right) .
$$

Hence we have

$$
(a, k) \equiv(b, k) \quad\left(\bmod G\left(A_{k} / K\right)\right) .
$$

This is equivalent to $a \equiv b\left(\bmod N_{G} A(K)\right)$, and this implies $f_{K / k} \sim g_{K / k}$. Then as in Lemma 1 we have $f_{K / k} \sim g_{K / k}$ for every normal extension $K / k$.

(ii) We shall prove next the existence of homomorphisms $\Phi_{K, k}$ satisfying $\mathrm{A}^{*}, \mathrm{~B}^{*}, \mathrm{C}^{*}$. By (i) we may take a frame $\Sigma_{K / k}^{\prime}=\left(\left\{\boldsymbol{u}_{\sigma}^{\prime}\right\},\left\{\boldsymbol{f}_{\boldsymbol{K} / k}\right.\right.$ $(\sigma, \tau)\})$ in each $W^{\prime}(K / k)$. Moreover, it is easy to see that for given $\{K, \ell, k\}$ (in case II) we can choose frames $\Sigma_{K / k}^{\prime}$ and $\Sigma_{K / \ell}^{\prime}$ such that the homomorphisms $\nu_{K, \xi, k}^{\prime}$ is defined as in the proof (iii) of Theorem 1 ; and similarly for $\rho^{\prime}$ in each $\{L, K, k\}$ in case I. But what we want to prove is that we can choose a frame $\nu_{K / k}^{\prime}$ of $W(K / k)$ once for all such that $\nu^{\prime}$ and $\rho^{\prime}$ are expressed by these frames as in the proof of Theorem 1. For this purpose let $k_{0} \subset K_{1} \subset \cdots \subset K_{n} \subset \cdots\left(K_{n} / k_{0}\right.$ : normal) and $\boldsymbol{\Omega}=\bigcup K_{n}$ and let us choose the frames $\Sigma_{K_{1} / k_{0}}^{\prime}, \Sigma_{K_{2} / k_{0}}^{\prime}, \cdots$ successively such that the frames $\Sigma_{K_{n} / k_{0}}^{\prime}$ and $\Sigma_{K_{n-1} / k_{0}}^{\prime}$ are in the required relation with respect to $\rho^{\prime}$ for $\left\{K_{n}, K_{n-1}, k_{0}\right\}$. Then we take the frame $\Sigma_{K, k}^{\prime}$ for $k_{0} \subset k \subset K \subset K_{n}$ using the relations $\nu$ and $\rho$ for $\left\{K_{n}, k, k_{0}\right\}$ and $\left\{K_{n}, K, k\right\}$ respectively. That this choice of the frame $\Sigma_{K / k}^{\prime}$ is independent of $K_{n}$ and that $\rho^{\prime}$ and $\nu^{\prime}$ are expressed just as in the proof of Theorem 1 by means of these frames can be proved without difflculties. So we omit the details here. After choosing these frames $\Sigma_{K(k}^{\prime}$ in $W^{\prime}(K / k)$ it is easy to define the isomorphism $\Phi_{K, k}: W(K / k) \rightarrow W^{\prime}(K / k)$, namely let

$$
W(K / k)=\bigcup_{\sigma \mathrm{e} G} A(K)^{*} \cdot u_{\sigma}, u_{\sigma} \cdot u_{\tau}=f_{K / k}(\sigma, \tau)^{*} \cdot u_{\sigma \tau}
$$




$$
W(K / k)=\bigcup_{\sigma \mathrm{e} G} A(K)^{\prime} \cdot u_{\sigma}^{\prime}, u_{\sigma}^{\prime} \cdot u_{\tau}^{\prime}=f_{K k}(\sigma, \tau)^{\prime} \cdot u_{\sigma \tau}^{\prime}
$$

we define then

$$
\Phi_{K, k}\left(a^{*}\right)=a^{\prime}(a \in A(K)), \Phi_{K, k}\left(u_{\sigma}\right)=u_{\sigma}^{\prime} .
$$

It is evident that $\mathrm{A}^{*}, \mathrm{~B}^{*}, \mathrm{C}^{*}$ hold for these $\Phi_{K, k^{*}}$

(iii) That these $\Phi_{K, k}$ satisfy $\mathrm{A}^{\prime *}$ follows from the following Lemma :

LEMMA 3. If a system of groups $\{W(K / k)\}$ satisfies the conditions $\mathrm{A}, \mathrm{B}, \mathrm{C}, \mathrm{A}^{\prime}, \mathrm{B}^{\prime}, \mathrm{C}^{\prime}$ then the homomorphisms $\xi_{K, k}$ are determined uniquely by other homomorphisms $\eta, \lambda, \mu, \nu, \rho, \pi$.

(PROOF) Let $\xi_{K, k}$ and $\xi_{K, k}^{\prime}$ be two homomorphisms satisfying the conditions $\mathrm{A}^{\prime}, \mathrm{B}^{\prime}, \mathrm{C}^{\prime}$, while the other homomorphisms $\lambda, \pi, \mu, \nu, \rho, \eta$ are the same for $\xi$ and $\xi^{\prime}$. If we use the same notations as in the proof of Theorem 1 we have

$$
\left\{\begin{array}{l}
\xi_{K, k}^{\prime}\left(u_{\sigma}\right)=\xi_{K, k}\left(u_{\sigma}\right) \cdot B_{K}(\sigma) \quad B_{K}(\sigma) \in \Gamma(K) \\
B_{K}(\sigma) \cdot B_{K}(\tau)^{\sigma} \cdot B_{K}(\sigma \tau)^{-1}=1
\end{array}\right.
$$

By the considerations and notations in (vii) of the proof of Theorem 1 it follows from the condition $\mathrm{C}^{\prime}$ for $k \subset K \subset L$ that

$$
\psi B_{L}(\bar{\sigma})=B_{K}(\widetilde{\sigma})
$$

where $\psi=\psi\left(I^{\prime}(K), \Gamma(L / K)\right)$. In particular, if we take $k \subset K \subset L \subset A_{K}$ then $\Gamma(L / K)=\Gamma^{\top}(K)$ and $\psi$ is the identity. Hence (48) implies that $B_{K}(\tilde{\sigma})(\tilde{\sigma} \in G(K / k))$ belong to $G\left(A_{K} / L\right)$. Since $L$ is an arbitrary intermediate field in $A_{K} / K$ we have $B_{K}(\tilde{\sigma})=1(\tilde{\sigma} \in G(K / k))$, namely $\xi_{K, k}^{\prime}=$ $\xi_{K, k}$, q. e.d.

THEOREM 3. Let $B(K)$ be the kernel of $\eta_{K}: A(K) \rightarrow \Gamma(K)$ and let us assume that

$$
H^{1}(G, B(K))=0 \text {. }
$$

Then the isomorphism $\Phi_{K, k}$ in Theorem 2 is determined up to the inner automorphism by an element of $\lambda_{K, k} B(K)$.

(ProOF) Let us assume that $\lambda=\lambda^{\prime}, \pi=\pi^{\prime}$, etc. in $\mathrm{A}^{*}, \mathrm{~B}^{*}, \mathrm{C}^{*}, \mathrm{~A}^{\prime *}$. Let us fix a frame $\Sigma_{K, k}=\left(\left\{u_{\sigma}\right\},\left\{f_{K k}\right\}\right)$ of $W(K / k)$ :

$$
W(K / k)=\bigcup_{\sigma \mathrm{e} G} A(K)^{*} \cdot u_{\sigma}, \quad u_{\sigma} \cdot u_{\tau}=f_{K / k}(\sigma, \tau)^{*} \cdot u_{\sigma \tau} \cdot
$$


Then $\Phi_{K, k}$ is determined by $\Phi_{K, k}\left(a^{*}\right)=a^{*}$ and $\Phi_{K, k}\left(u_{\sigma}\right)=u_{\sigma}^{\prime}$. By conditions $\mathrm{A}, \mathrm{A}^{\prime}$ we have

$$
u_{\sigma}^{\prime} \cdot u_{\tau}^{\prime}=f_{K / k}(\sigma, \tau)^{*} u_{\sigma \tau}^{\prime}, \xi_{K, k}\left(u_{\sigma}\right)=\xi_{K, k}\left(u_{\sigma}^{\prime}\right)
$$

Hence we have

$$
u_{\sigma}^{\prime}=b_{\sigma}^{*} \cdot u_{\sigma},\left(b_{\sigma} \in A(K)\right), b_{\sigma} \cdot b_{\tau}^{\sigma} \cdot b_{\sigma \tau}^{-1}=1 \text {, and } \eta_{K}\left(b_{\sigma}\right)=1 \text {. }
$$

Therefore, we have $b_{\sigma} \in B(K)$. Since $H^{1}(G, B(K))=0$ we can find an element $c \in B(K)$ such thet $b_{\sigma}=c^{1-\sigma}(\sigma \in G)$ hold. Then we have $u_{\sigma}^{\prime}=$ $c^{1-\sigma} u_{\sigma}$ and hence

$$
\Phi_{K, k}\left(a^{*} u_{\sigma}\right)=a^{*}\left(c^{1-\sigma}\right)^{*} \cdot u_{\sigma}=c^{*}\left(a^{*} \cdot u_{\sigma}\right) c^{*-1} .
$$

Conversely the mapping (50) satisfies all the conditions $\mathrm{A}^{*}, \mathrm{~B}^{*}, \mathrm{C}^{*}$, $\mathrm{A}^{\prime *}$, q. e. d.

REMARK. We omit the discussion on the uniqueness property of a (not necessarily strong) system of Weil groups.

\section{§3. Strong system of generalized Weil groups}

1. Let $\{\Omega, A(K)\}$ be a class formation and let $\{W(K / k)\}$ be a strong system of Weil groups associated with it. We shall prove the following existence theorem of generalized Weil groups:

THEOREM 4. Let us assume the axiom $\mathrm{P}^{\prime}$ :

$$
\Omega=\bigcup_{n} K_{n} \quad\left(k_{0} \subset K_{1} \subset K_{2} \subset \cdots \subset K_{n} \subset \cdots \subset \Omega\right)
$$

in $\Re$. Then there exists a system of groups $\{W(k) ; k \in \Re\}$ with the following properties:

$\left(\mathrm{A}_{1}\right)$ Let $G(k)$ be the compact Galois group of $\Omega / k$ (i. e. $G(k)=$ $G(\Omega / k))$. There exists an into-homomorphism

$$
\pi_{k}: W(k) \rightarrow G(k)
$$

whose image $\pi_{k} W(k)$ is dense in $G(k)$, and an onto-homomorphism

$$
\mu_{k}: W(k) \rightarrow A(k) \text {. }
$$

We denote its kernel by $W(k)^{c c}$ such that

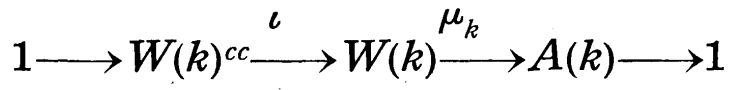


is an exact sequence.

$\left(\mathrm{A}_{2}\right)$. The diagram

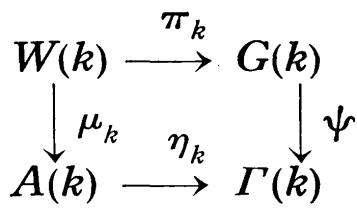

is commutative.

$\left(\mathrm{B}_{1}\right) \quad$ For $k \subset l(k, \mathfrak{l} \in \Re)$ there exists an into-isomorphism

$$
\nu_{\imath, k}: W(\ell) \rightarrow W(k)
$$

such that

$$
\underset{\downarrow}{W(l) \stackrel{\pi_{l}}{\longrightarrow}} G(\mathfrak{l})
$$

is a commutative diagram. We have also

$$
\nu_{i, k} W(t)=\pi_{k}^{-1} G(l) .
$$

$\left(\mathrm{B}_{2}\right) \quad$ For $k \subset l \subset j$ the transitivity relation

$$
\nu_{\boldsymbol{Z}, k}{ }^{\nu_{j, \boldsymbol{z}}}=\boldsymbol{\nu}_{j, k}
$$

holds.

$\left(\mathrm{C}_{1}\right)$ For a normal extension $K / k(k, K \in \Re)$ there exists an ontohomomorphism

$$
\rho_{K, k}: W(k) \rightarrow W(K / k)
$$

such that
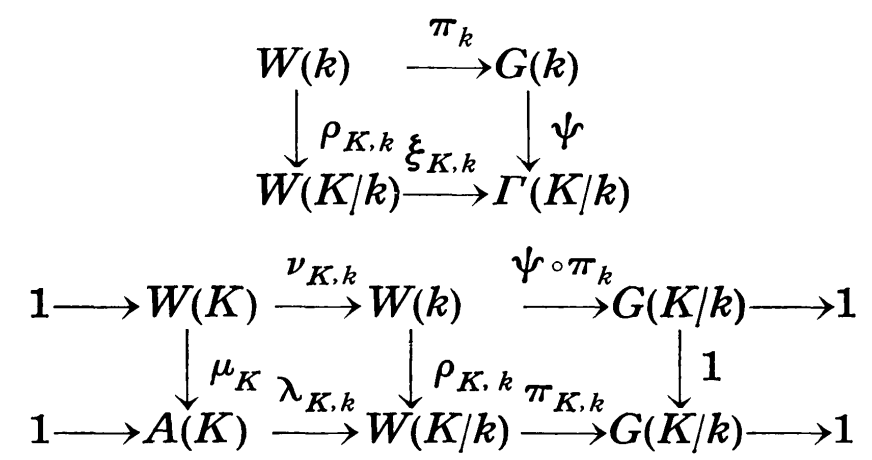
are commutative diagrams. Here $\psi=\psi(G(K / k), G(k))$. We remark here that from (12) follows

$\left(\mathrm{C}_{2}\right)$ In the same case

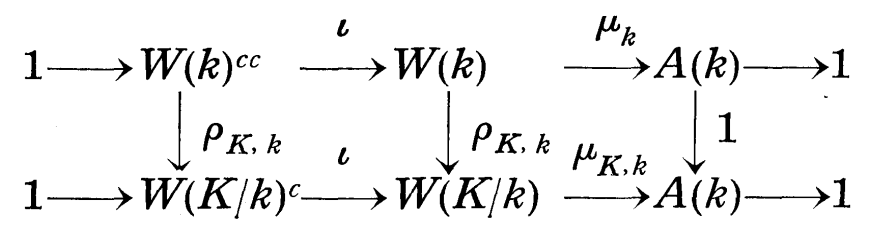

is a commutative diagram. We remark here that from (11), (14) and $\S 2$, (17) follows (5).

$\left(\mathrm{C}_{3}\right)$ Let $k \subset K \subset L$ and $K / k, L / k$ be both normal. Then the transitivity relation

$$
\rho_{L, K, k} \circ \rho_{L, k}=\rho_{K, k}
$$

holds.

$\left(\mathrm{C}_{4}\right) \quad$ Let $k \subset l \subset K$ and $K / k$ be normal. Then

$$
\rho_{K, k^{\circ} \nu_{l, k}=\nu_{K, l, k} \circ \rho_{K, l} .}
$$

We call this system $\{W(k) ; k \in \Re\}$ a strong system of generalized Weil groups associated with $\{\Re, A(K), W(K / k)\}$.

(PROOF) (i) Let $k \in \Re . \quad$ By (1) $k \subset K_{r}$ for a certain $r$. In the following we assume that the fields $\left\{K_{n}\right\}$ in (1) are all normal over $k_{0}$. Then we consider a sequence of groups $\left\{W\left(K_{n} / k\right) ; n=r, r+1, \cdots\right\}$ and a set of onto-homomorphisms

$$
\Pi_{n}=\rho\left(K_{n}, K_{n-1}, k\right): W\left(K_{n} / k\right) \rightarrow W\left(K_{n-1} / k\right) \quad(n=r+1, r+2, \cdots) .
$$

These determine the inverse limit group $W(k)$ :

$$
W(k)=\lim _{n \rightarrow \infty}\left\{W\left(K_{n} \mid k\right), \Pi_{n}\right\} \text {. }
$$

Since all $\Pi_{n}$ are onto-homomorphisms we can define naturally the onto-homomorphism

$$
\rho\left(K_{n}, k\right): W(k) \rightarrow W\left(K_{n} / k\right) \quad\left(k \subset K_{n}\right)^{8)}
$$

such that $\rho\left(K_{n-1}, K_{n}, k\right) \circ \rho\left(K_{n-1}, k\right)=\rho\left(K_{n}, k\right)$ holds. On the other hand

8) We also use the notations $\rho(K, k)$ instead of $\rho_{K, k}$ etc. 
the Galois group $G(k)=G(\Omega / k)$ can be considered also as the inverse limit group of the system $\left\{G\left(K_{n} / k\right), \psi\left(G\left(K_{n} / k\right), G\left(K_{n+} / k\right)\right)\right\}$. Since the following diagram is commutative:

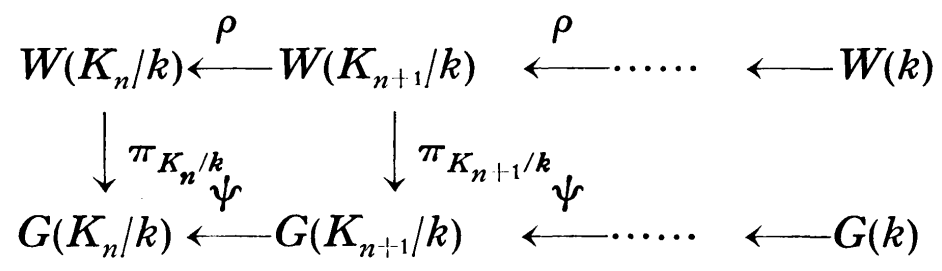

we can define the homomorphism $\pi_{k}: W(k) \rightarrow G(k)$ by

$$
\pi_{k}(u)=\lim \left(\pi\left(K_{n}, k\right) \circ \rho\left(K_{n}, k\right)(u)\right) \quad u \in W(k) \text {. }
$$

Since each $\pi\left(K_{n} / k\right)$ is an onto-homomorphism the image $\pi_{k} W(k)$ is dense in $G(k)$.

(ii) Consider the following commutative diagram:

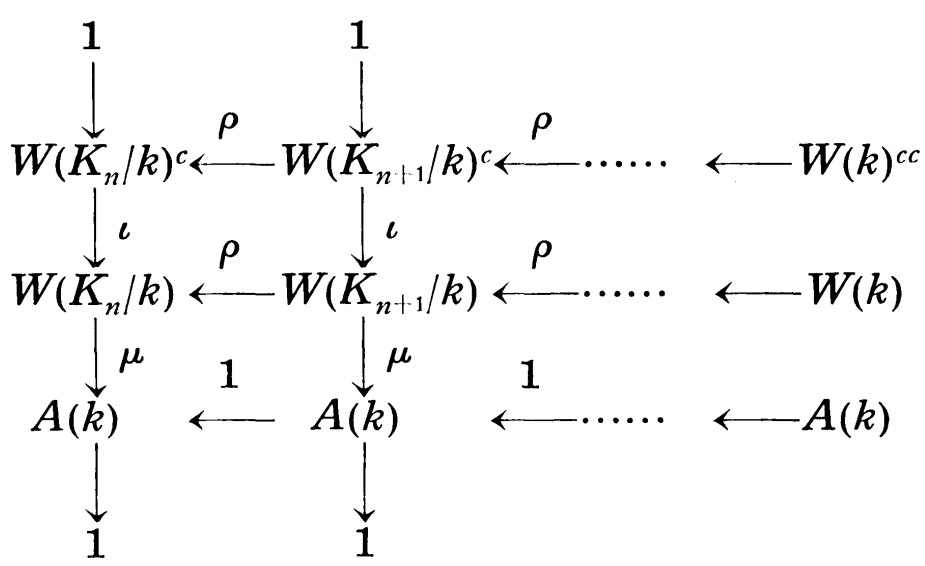

where each column is exact. Then we can define the homomorphism $\mu_{k}: W(k) \rightarrow A(k)$ by

$$
\mu_{k}(u)=\lim \left(\mu\left(K_{n}, k\right) \circ \rho\left(K_{n}, k\right)(u)\right) \in A(k) \quad u \in W(k) .
$$

Since $\rho\left(K_{n}, k\right)$ is an onto-homomorphism $\mu_{k}$ is also an onto-homomorphism. Here the kernel of $\mu_{k}$ is the limit group of $\left\{W\left(K_{n} / k\right)^{c}\right\}$ and contains the commutator group of $W(k)$.

(iii) Let $k \subset l$ and

$$
W(\mathfrak{l})=\lim \left\{W\left(K_{n} / \mathfrak{l}\right), \rho\left(K_{n+1}, K_{n}, \mathfrak{l}\right)\right\}, W(k)=\lim \left\{W\left(K_{n} / k\right), \rho\left(K_{n+1}, K_{n}, k\right)\right\} .
$$

Then from the following commutative diagram 


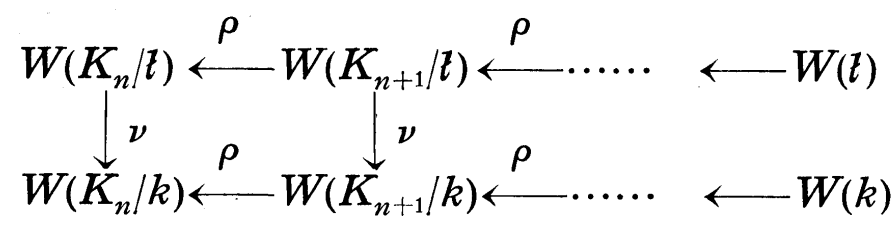

it follows that we can define the homomorphism $\nu_{z ; k}: W(\ell) \rightarrow W(k)$ by

$$
\nu_{\imath ; k}(u)=\lim \left(\nu\left(K_{n}, \ell, k\right) \circ \rho\left(K_{n}, l\right)(u)\right) \quad u \in W(\ell) .
$$

That $\nu_{i ; k}$ is an into-isomorphism follows from the fact that each $\nu\left(K_{n}, l, k\right)$ is an into-isomorphism. The commutativity of (7) follows from the following commutative diagram and the definition of $\pi_{k}$ and $\nu_{z ; k}$ :

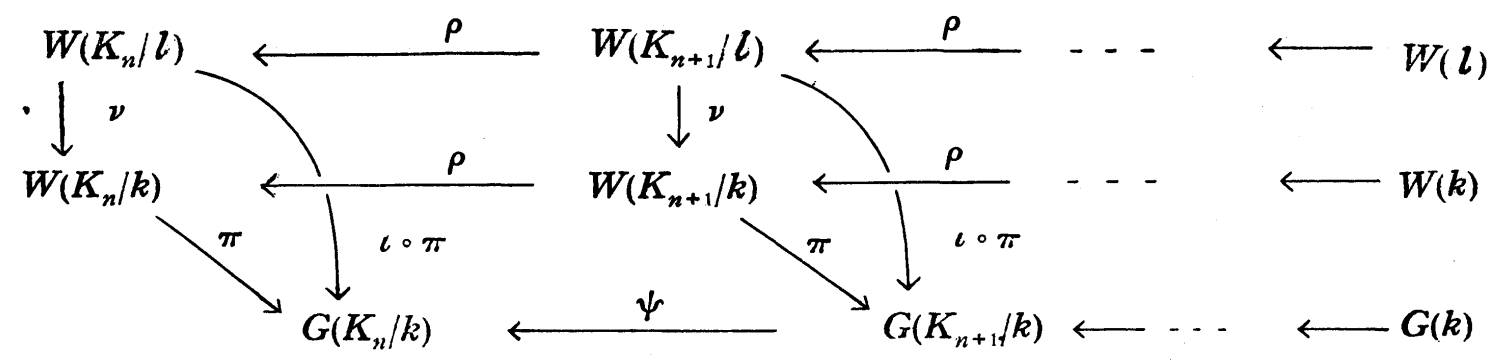

$\left(\mathrm{B}_{2}\right)$ can be proved similarly.

(iv) Let $k \subset K \subset K_{n}(n=r, r+1, \cdots)$. Then using the commutative diagram:

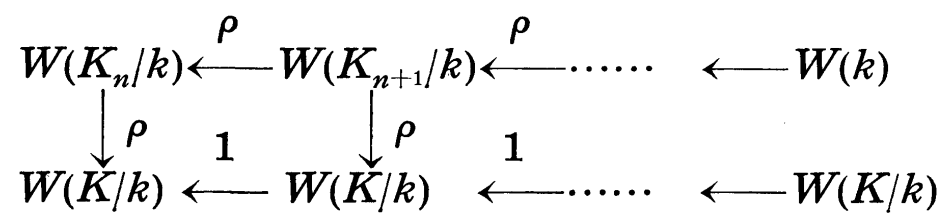

we define the homomorphism $\rho_{K ; k}: W(k) \rightarrow W(K / k)$ by

$$
\rho_{K, k}(u)=\rho\left(K_{n}, K, k\right) \circ \rho\left(K_{n}, k\right)(u) \quad u \in W(k) .
$$

Since $\rho\left(K_{n}, K, k\right)$ and $\rho\left(K_{n}, k\right)$ are onto-homomorphisms $\rho_{K, k}$ is an ontohomomorphism. Now we can verify $\left(\mathrm{C}_{1}\right),\left(\mathrm{C}_{2}\right),\left(\mathrm{C}_{3}\right)\left(\mathrm{C}_{4}\right)$ as above using corresponding diagrams. It is evident that the definition of $W(k), \rho, \pi, \nu$, does not depend on the choice of the sequence $\left\{K_{n}\right\}$, q.e.d.

2. Let $B(K)$ be the kernel of $\eta_{K}: A(K) \rightarrow \Gamma(K)$. Let $\left\{K_{n}\right\}$ be the sequence in (1) and let us put 
(24) $\quad \Pi_{n+1}^{\prime}=\varphi\left(K_{n}, K_{n+1}\right)^{-1} \cdot N_{G\left(K_{n+1} / K_{n}\right)}: B\left(K_{n+1}\right) \rightarrow B\left(K_{n}\right) \quad(n=1,2, \cdots)$.

Then we can define the inverse limit group $B$ by

$$
B=\lim \left\{B\left(K_{n}\right), \Pi_{n}^{\prime}\right\} \text {. }
$$

Let us denote the canonical homomorphism by $\psi_{n}: B \rightarrow B\left(K_{n}\right)$ so that

$$
\psi_{n}=\Pi_{n+1}^{\prime} \circ \psi_{n+1} \text {. }
$$

THEOREM 5. Let us assume, furthermore, that (i) for each $k \in \Omega$

$$
\eta_{K}(A(k))=\Gamma(k)
$$

holds, and (ii) for each normal $K / k$ with $G=G(K / k)$

(27)

$$
\boldsymbol{\varphi}_{K, k} B(k)=N_{G} B(K)
$$

holds. Then we have stronger results than in Theorem 4:

$\left(\mathrm{A}_{1}\right)$ There exists

$$
\text { an into-isomorphism } \lambda_{k}: B \rightarrow W(k)
$$

such that

$$
1 \longrightarrow B \stackrel{\lambda_{k}}{\longrightarrow} W(k) \stackrel{\pi_{k}}{\longrightarrow} G(k) \longrightarrow 1
$$

is exact.

$\left(\mathrm{A}_{2}\right)$ Let $C(k)$ be the kernel of $\psi_{k}: B \rightarrow B(k)$. Instead of (5) we have the following commutative diagram where each column and each row are exact sequences:

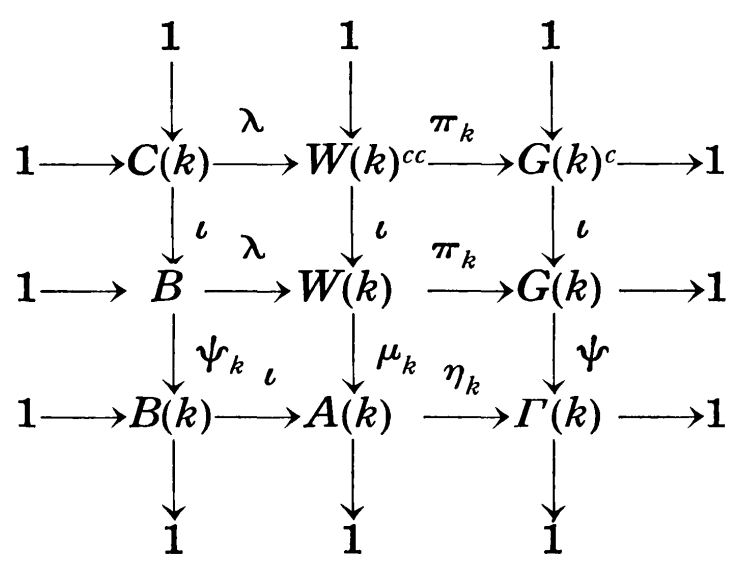

where $\psi_{k}$ is defined naturally by the definition of $B$ in (25). 
$\left(\mathrm{C}_{1}\right)^{\prime} \quad$ Instead of $(11)$ we have the following commutative diagram, where each column and each row are exact sequences:

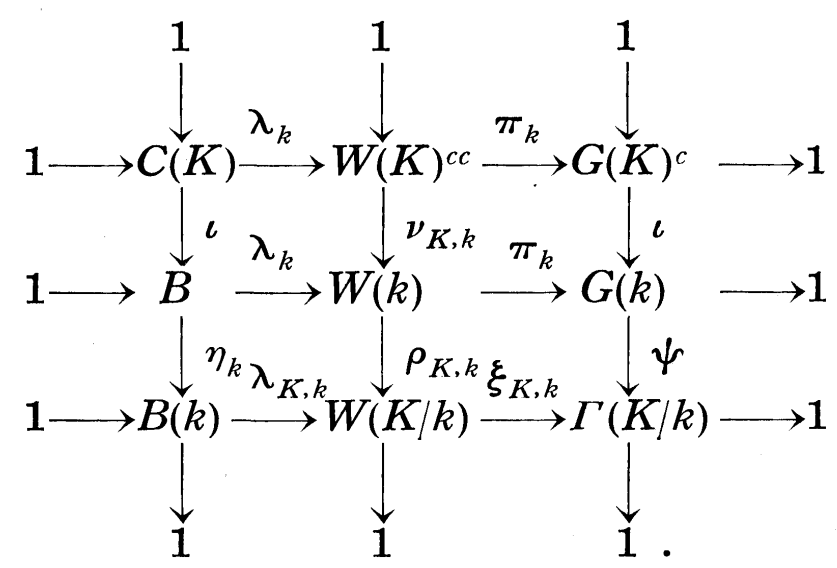

(ProOF) By our assumption (27)] $\Pi_{n}^{\prime}(n=1,2, \ldots)$ are onto-homomorphisms and hence $\psi_{n}: B \rightarrow B\left(K_{n}\right)$ are also onto-homomorphisms. Since the following is a commutative diagram:

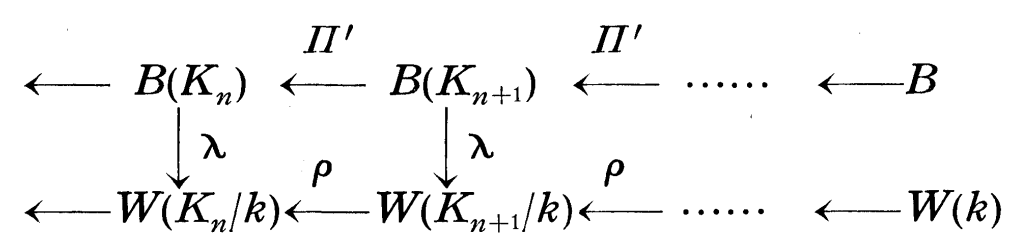

we can define $\lambda_{k}: B \rightarrow W(k)$ by

$$
\lambda_{k}(b)=\lim \left(\lambda\left(K_{n}, k\right) \circ \psi_{n}(b)\right) \quad b \in B .
$$

Since $\lambda\left(K_{n}, k\right): B\left(K_{n}\right) \rightarrow W\left(K_{n} / k\right)$ are all into-isomorphisms, $\lambda_{k}$ is also an into-isomorphism. Next consider the following commutative diagram:

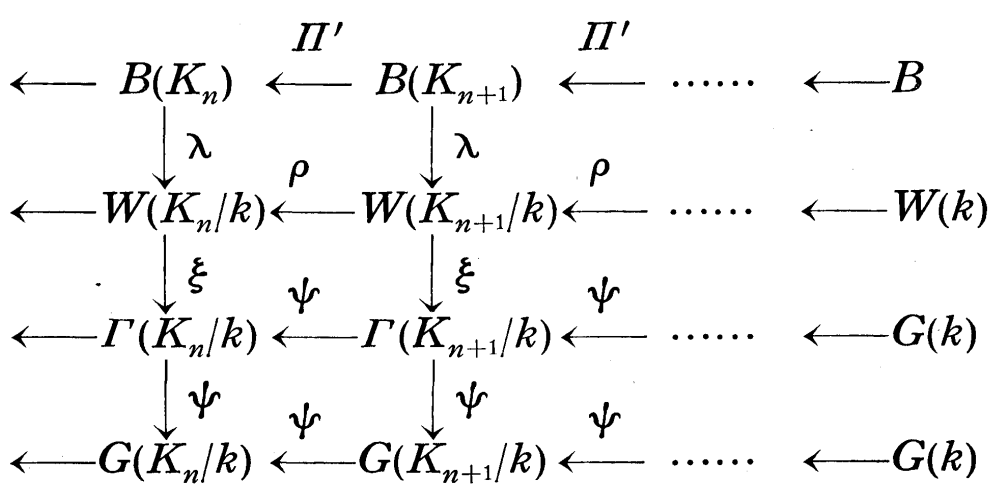


Here we have $G(k)=\lim \left(\Gamma\left(K_{n} / k\right), \psi\right)=\lim \left(G\left(K_{n} / k\right), \psi\right)$. For $\sigma \in G(k)$ let us denote $\sigma_{n}=\psi\left(G\left(K_{n} / k\right), G(k)\right)(\sigma) \in G\left(K_{n} / k\right)$ and $\rho_{n}=\psi\left(\Gamma\left(K_{n} / k\right)\right.$, $G(k))(\sigma)$ so that $\sigma=\lim \sigma_{n}=\lim \rho_{n}$. Now take arbitrarily $\alpha_{n} \in W\left(K_{n} / k\right)$ such that $\xi\left(K_{n}, k\right)\left(\alpha_{n}\right)=\rho_{n}$ holds. Then by means of $\Pi_{n+1}^{\prime} B\left(K_{n+1}\right)=$ $B\left(K_{n}\right)$ and Kernel $\xi\left(K_{n} / k\right)=\lambda B\left(K_{n}\right)$ we can find an element $\alpha_{n+1} \in$ $W\left(K_{n+1} / k\right)$ such that

$$
\alpha_{n}=\rho\left(K_{n+1}, K_{n}\right) \alpha_{n+1}, \quad \rho_{n+1}=\xi\left(K_{n+1}, k\right) \alpha_{n+1}
$$

holds. (Precisely, take $\beta_{n+1} \in W\left(K_{n+1} / k\right)$ such that $\xi\left(K_{n+1}, k\right) \beta_{n+1}=\rho_{n+1}$ holds. Then its image $\beta_{n}=\rho\left(K_{n+1}, K_{n}, k\right) \beta_{n+1}$ satsifies $\xi\left(K_{n} / k\right) \alpha_{n} \bullet \beta_{n}^{-1}=1$ by the commutativity of (33). Hence $\alpha_{n} \bullet \beta_{n}^{-1} \in \lambda\left(K_{n}, k\right) B\left(K_{n}\right)$. So take $\gamma_{n+1} \in B\left(K_{n+1}\right)$ such that $\alpha_{n} \bullet \beta_{n}^{-1}=\lambda\left(K_{n}, k\right) \circ \Pi_{n}^{\prime}\left(\gamma_{n+1}\right)$ holds. Let us put $\alpha_{n+1}=\lambda\left(K_{n+1}, k\right)\left(\gamma_{n+1}\right) \bullet \beta_{n+1}$. Then this element $\alpha_{n+1}$ satisfies (34)). Continuing this process we can find a sequence $\left\{\alpha_{n} \in W\left(K_{n} / k\right)\right\}$ such that $\alpha=\lim \alpha_{n} \in W(k)$ satisfies $\pi_{k}(\alpha)=\sigma$. Hence we have $\pi_{k} W(k)=$ $G(k)$. Finally the whole exactness of the sequence (29) follows also from the commutativity of the diagram (33).

The commutativity of the diagrams [(30), (31) can be proved in similar manner, q. e. d.

REMARK 1. As to the uniqueness theorem for generalized Weil groups satisfying all the formaulas $\left(A_{1}\right),\left(A_{2}\right),\left(B_{1}\right),\left(B_{2}\right),\left(C_{1}\right),\left(C_{2}\right),\left(C_{3}\right)$, $\left(\mathrm{C}_{4}\right),\left(\mathrm{A}_{1}\right)^{\prime},\left(\mathrm{A}_{2}\right)^{\prime},\left(\mathrm{C}_{1}\right)^{\prime}$ we can prove the analogous theorem as Theorem 2 under the assumption $\mathrm{P}, \mathrm{P}^{\prime}$ and (i), (ii) in Theorem 5 .

REMARK 2. It is an open question whether every system of Weil groups $\{W(K / k)\}$ is a strong system or not.

REMARK 3. We don't discuss here the problem to construct a system of generalized Weil groups from a (not necessarily strong) system of Weil groups $\{W(K / k)\}$.

\section{$\S 4$. System of Weil groups in algebraic function fields}

1. Let $k_{0}$ be an algebraic function field in one variable over the complex number field $C$ and $\boldsymbol{\Omega}$ be the maximal unramified extension over $k_{0}$. As we have proved (Kawada-Tate [8]) we can define a class formation $\{\Omega, A(K)\}$ as follows. Let $K \in \Re, D(K)$ be the group of all divisors of $K, E(K)$ be the group of all divisor classes of $K$. Let us introduce a locally compact topology in $E(K)$ such that the 
subgroup $E_{0}(K)$ of all divisor classes of degree 0 is compact and $E(K) / E_{0}(K)$ is discrete. Consider the character group $E(K)^{\wedge}$ (in the sense of Pontrjagin). Put $A(K)=E(K)^{\wedge}$. For $k \subset K(k, K \in \Omega)$ we define $\varphi_{K: k}: E(k)^{\wedge} \rightarrow E(K)^{\wedge}$ by conorm. Then we can prove that $\{\Omega$, $A(K)\}$ is a class formation.

Let $R(K)$ be the Riemann surface of $K, F(K)$ be the fundamental group of $R(K), H(K)$ be the one-dimensional homology group of $R(K)$ with integral coefficients. We can identify $H(K)=F(K) / F(K)^{c}$. We denote by $\Gamma$ a closed curve on $R(K)$ (with initial point $\mathfrak{F}_{0}$ ), by $\bar{\Gamma}$ its homotopy class (so an element of $F(K)$ ), by $\tilde{\Gamma}$ its homology class. We use the additive notation for chains and the multiplicative notation in $F(K)$. Let $\mathfrak{A}=\prod_{i} \mathfrak{P}_{i}^{\nu_{i}}$ be a divisor of degree 0 on $R(K)$ and $d \log \mathfrak{A}$ be the differential of the 3rd kind on $R(K)$ such that (i) at each point $\mathfrak{B}_{i} d \log \mathfrak{A}$ has a simple pole with residue $\nu_{i}$, (ii) all periods of $d \log \mathfrak{A}$ are pure imaginary. If $B$ is a 1-chain on $R(K)$ we define

$$
[B, \mathfrak{A}]=\int_{B} d \log \mathfrak{A}, \quad(B, \mathfrak{A})=\exp [B, \mathfrak{A}] .
$$

Let us fix a prime divisor $\mathfrak{P} . \quad \chi \in E(K)^{\wedge}$ induces a character on $D(K)$ for which we use the same notation: $\chi(\mathfrak{H})=\chi(\hat{\mathfrak{U}})$. Here by $\widetilde{\mathfrak{A}}$ we mean the divisor class containing a divisor $\mathfrak{A}$. Then $\chi$ can be expressed in the form

$$
\chi\left(\mathfrak{A P} \beta^{*}\right)=\left(I^{r}, \mathfrak{U}\right) \cdot \lambda^{s} \quad \mathfrak{A} \in E_{0}(K)
$$

by a 1-cycle $\Gamma$ and a complex number $\lambda$ with absolute value 1 . Here $\Gamma$ is uniquely determined up to boundaries. We denote then $\chi=\chi(\widetilde{\Gamma}, \lambda)$.

Let $K / k$ be a finite unramified extension. We denote be $\pi=\pi_{K / k}$ the projection of $R(K)$ onto $R(k)$ and by $V=V_{K \mid k}$ the transfer mapping: $H(k) \rightarrow H(K)$. Then we have

$$
\varphi_{K ; k} \chi_{k}(\widetilde{\gamma}, \lambda)=\chi_{K}(V \widetilde{\gamma}, \lambda) \quad \hat{\gamma} \in H(k) .
$$

Let $K / k$ be normal with the Galois group $G=G(K / k)$. We can verify

$$
\chi(\hat{\Gamma}, \lambda)^{\sigma}=\chi\left(\sigma \widehat{\Gamma}, \lambda \cdot\left(\Gamma, \mathfrak{P}^{\sigma^{-1}}\right)\right) .
$$

In the following we use the notation 


$$
Q[\sigma, B]=\Im\left[B, \mathfrak{P}^{\sigma}{ }^{-1}\right], Q(\sigma, B)=\exp Q[\sigma, B]
$$

for $\sigma \in G$ and for 1-chain $B$, where $\Im$ denote the imaginary part of a complex number. Let $F(k)=\bigcup_{\sigma \in G} \pi F(K) \cdot \beta_{\sigma}$ and $\beta_{\sigma} \cdot \pi \bar{\Gamma} \cdot \beta_{\sigma}^{-1}=\pi(\sigma \bar{\Gamma})$. Choose a curve $B_{\sigma}$ on $R(K)$ such that $B_{\sigma}$ connects $\mathfrak{P}_{0}$ with $\sigma \mathfrak{P}_{0}$ and $\pi B_{\sigma}$ belongs to $\beta_{\sigma}$. Let then $\Gamma^{\top}(\sigma, \tau)=B_{\sigma}+\left(\sigma B_{\tau}\right)-B_{\sigma \tau}$ and

$$
\lambda(\sigma, \tau)=Q\left(\sigma, B_{\tau}\right) \text {. }
$$

Then

$$
f_{K / k}(\sigma, \tau)=\chi(\widetilde{\Gamma}(\sigma, \tau), \lambda(\sigma, \tau))
$$

satisfies all the properties of a fundamental 2-cocycle. We use later the following relations

$$
\left\{\begin{array}{l}
Q[\tau \sigma, \Gamma]=Q[\sigma, \Gamma]+Q[\tau, \sigma \Gamma] \\
Q[\rho, \Gamma(\sigma, \tau)]=Q\left[\rho, B_{\sigma}\right]-Q\left[\sigma, B_{\tau}\right]+Q\left[\rho \sigma, B_{\tau}\right]-Q\left[\rho, B_{\sigma \tau}\right]
\end{array}\right.
$$

which can be verified easily.

2. We shall consider next a system of Weil groups in algebraic function fields. For normal $K / k(k, K \in \Re)$ we can express $W(K / k)$ by

$$
\left\{\begin{array}{l}
W(K / k)=\bigcup_{\sigma \in G} A(K)^{*} \cdot u_{\sigma} \quad\left(A(K)=E(K)^{\wedge}\right) \\
u_{\sigma} \cdot \chi(\widetilde{\Gamma}, \lambda)=\chi\left(\sigma \widetilde{\Gamma}, \dot{\lambda} \cdot Q_{K / k}(\sigma, \Gamma)\right)^{*} \cdot u_{\sigma} \\
u_{\sigma} \cdot u_{\tau}=\chi(\widetilde{\Gamma}(\sigma, \tau), \lambda(\sigma, \tau))^{*} \cdot u_{\sigma \tau}
\end{array}\right.
$$

From (9) follows that

$$
T^{*}=\left\{\chi(0, \lambda)^{*}\right\}
$$

belongs to the centre of $W(K / k)$. We identify $\chi(0, \lambda)$ with $\lambda$ and we denote $T=\{\lambda ; \lambda \in C,|\lambda|=1\}$. Let

$$
J(K / k)=F(k) / \pi F(K)^{c}
$$

Then we have

$$
\begin{aligned}
& J(K / k)=\bigcup_{\sigma \in G} H(K) \cdot \widetilde{\beta}_{\sigma} \\
& \widetilde{\beta}_{\sigma} \cdot \widetilde{\beta}_{\tau}=\widetilde{\Gamma}(\sigma, \tau) \widetilde{\beta}_{\sigma \tau}
\end{aligned}
$$

Hence if we put 


$$
\pi_{K \mid k}^{\prime}\left(u_{\sigma}\right)=\beta_{\sigma}, \pi_{K \mid k}^{\prime}(\chi(\widetilde{\Gamma}, \lambda))=\pi_{K \mid k} \tilde{\Gamma},
$$

we have an exact sequence

$$
1 \longrightarrow T \stackrel{*}{\longrightarrow} W(K / k) \stackrel{\pi^{\prime}}{\longrightarrow} J(K / k) \longrightarrow 1 .
$$

For $\alpha \in F(k)$ let us denote by $\alpha^{*}$ the class of $\alpha \bmod \pi F(K)^{c}$ (i.e. $\alpha^{*} \in J(K / k)$ ), and let us choose a fixed representative path $\left[\alpha^{*}\right]$ on $R(K)$ starting from $\mathfrak{P}_{0}$ such that $\pi\left[\alpha^{*}\right]$ belongs to $\alpha$. In particular, we choose

$$
\left[\left(\pi \bar{\Gamma} \cdot \bar{\beta}_{\sigma}\right)\right]=\Gamma \cdot B_{\sigma},\left[(\pi \bar{\Gamma}(\sigma, \tau))^{*}\right]=B_{\sigma} \cdot \sigma B_{\tau} \cdot B_{\sigma \tau}^{-1} \cdot
$$

Then for new representatives

$$
U_{a^{*}}=\chi(\widetilde{\Gamma}, 1) \cdot u_{\sigma} \quad \text { for } \quad \alpha^{*}=\pi \bar{\Gamma} \cdot \bar{\beta}_{\sigma} \in J(K / k)
$$

we have

$$
\left\{\begin{array}{l}
W(K / k)=\underset{a^{*} \in J(K / k)}{\bigcup} T^{*} \cdot U_{a^{*}} \\
U_{a^{*}} \cdot \lambda^{*}=\lambda^{*} \cdot U_{a^{*}} \quad(\lambda \in T) \\
U_{a^{*}} \cdot U_{\beta^{*}}=a_{K \mid k}\left(\alpha^{*}, \beta^{*}\right) \cdot U_{a^{*} \beta^{*}}
\end{array}\right.
$$

where we put for $\alpha^{*}=\pi \bar{\Gamma} \cdot \bar{\beta}_{\sigma}$ and $\beta^{*}=\pi \bar{\Delta} \cdot \bar{\beta}_{\tau}$

$$
a_{K \mid k}\left(\alpha^{*}, \beta^{*}\right)=\lambda_{K \mid k}(\sigma, \tau) Q_{K \mid k}(\sigma, \Delta)=Q_{K \mid k}\left(\sigma,\left[\beta^{*}\right]\right) \text {. }
$$

Next we shall choose another system of representatives mod $T^{*}$, namely we put

$$
V_{a^{*}}=\exp \left(-Q_{K / k}\left[G,\left[\alpha^{*}\right]\right] / n\right) \cdot U_{a^{*}} .
$$

Then we have

$$
\left\{\begin{array}{l}
W(K / k)=\underset{a^{*} \in J(K \mid k)}{\bigcup} T^{*} \cdot V_{a^{*}} \\
\lambda^{*} \cdot V_{a^{*}}=V_{a^{*}} \cdot \lambda^{*} \quad(\lambda \in T) \\
V_{a^{*}} V_{\beta^{*}}=b_{K \mid k}\left(\alpha^{*}, \beta^{*}\right)^{*} \cdot V_{a^{*} \beta^{*}}
\end{array}\right.
$$

Using (8) $b\left(\alpha^{*}, \beta^{*}\right) \in T$ can be determined as follows: 


$$
\begin{aligned}
& b\left(\alpha^{*}, \beta^{*}\right)=a\left(\alpha^{*}, \beta^{*}\right) \cdot \exp \left(-\left\{Q\left[G,\left[\alpha^{*}\right]\right]+Q\left[G,\left[\beta^{*}\right]\right]-Q\left[G,\left[\alpha^{*} \beta^{*}\right]\right]\right\} / n\right) \\
& =\exp \left(Q[\sigma, \Delta]+Q\left[\sigma, B_{\tau}\right]-\left\{Q[G, \Gamma]+Q\left[G, B_{\sigma}\right]\right\} / n\right. \\
& \left.-\left\{Q[G, \Delta]+Q\left[G, B_{\tau}\right]\right\} / n+\left\{Q[G, \Gamma+\sigma \Delta+\Gamma(\sigma, \tau)]+Q\left[G, B_{\sigma \tau}\right]\right\} / n\right) \\
& =\exp (-\{Q[G, \Gamma]+Q[G, \sigma \Delta]+Q[G, \Gamma(\sigma, \tau)]-Q[G, \Gamma+\sigma \Delta+\Gamma(\sigma, \tau)]\} / n) \\
& =\exp \left(\left[-A, N_{G} \mathfrak{P} / \mathfrak{P}^{n}\right] / n\right)
\end{aligned}
$$

where $A=\Gamma+B_{\sigma}+\sigma \Delta+\sigma B_{\tau}-B_{\sigma \tau}-(\Gamma+\sigma \Delta+\Gamma(\sigma, \tau))=\left[\alpha^{*}\right]+\sigma\left[\beta^{*}\right]$ $-\left[\alpha^{*} \beta^{*}\right]$. On $R(K) \bar{A}$ belongs to $F(K)^{c}$. Hence 1-chain $A$ is homologous 0 on $R(K)$. Let us assume, for simplicity, a triangulation of $R(k)$ and $R(K)$ such that (i) $\mathfrak{P}_{0}$ is a vertex and $\mathfrak{P}$ is an inner point of a 2 -simplex, (ii) $\Gamma$ and $B_{\sigma}$ are all simplicial 1-chains, (iii) $\pi_{K \mid k}$ and $\sigma \in G$ are all simplicial mappings. Since $A$ is homologous 0 we can take a simplicial 2-chain $c^{2}$ such that $\partial c^{2}=\left[\alpha^{*}\right]+\sigma\left[\beta^{*}\right]-\left[\alpha^{*} \beta^{*}\right]$. Let us denote by $\operatorname{KI}\left(c^{2}, \mathfrak{D}\right)$ the Kronecker index of $c^{2}$ with respect to $\mathfrak{D}$ (i. e. the algebraic sum of the number of coverings of $\mathfrak{D}$ by each simplex in $\left.c^{2}\right)$ and by $\operatorname{LK}\left(\Gamma^{1}, \mathfrak{D}\right)=\operatorname{KI}\left(c^{2}, \mathfrak{D}\right)\left(\partial c^{2}=\Gamma^{1}\right)$ the linking coefficient. By the residue formula we have

$\left[\partial c^{2}, \mathfrak{P} / \mathfrak{Q}\right]=2 \pi i\left(\mathrm{KI}\left(c^{2}, \mathfrak{P}\right)-\mathrm{KI}\left(c^{2} \mathfrak{Q}\right)\right)$. Hence we have

$$
\begin{aligned}
b\left(\alpha^{*}, \beta^{*}\right) & =\exp \left(-\frac{2 \pi i}{n}\left\{(n-1) \mathrm{KI}\left(c^{2}, \mathfrak{P}\right)-\sum_{\tau \neq 1} \mathrm{KI}\left(c^{2}, \tau^{-1} \mathfrak{\beta}\right)\right\}\right) \\
& =\exp \left(\frac{2 \pi i}{n} \sum_{\tau \in} \mathrm{KI}\left(c^{2}, \tau \mathfrak{P}\right)\right)
\end{aligned}
$$

So finally

(20) $\quad b_{K \mid k}\left(\alpha^{*}, \beta^{*}\right)=\exp \left(\frac{2 \pi i}{n} \sum_{\tau \in G} \operatorname{LK}\left(\left[\alpha^{*}\right]+\left(\Psi \alpha^{*}\right)\left[\beta^{*}\right]-\left[\alpha^{*} \beta^{*}\right], \tau \mathfrak{P}\right)\right)$

where $\psi=\psi(G, J(K / k))$. By (20) we see that

$$
b_{K \mid k}\left(\alpha^{*}, \beta^{*}\right)^{n}=1 \quad(n=[K: k]) .
$$

We call the expression of $W(K / k)$ by (19) and (20) the standard expression of $W(K / k)$.

2. Here we shall find the explicit form of the homomorphisms $\lambda, \mu, \nu, \rho, \pi$ in Theorem 1 by means of the standard expression.

A. By the formaulas in proof (i) of Theorem 1 we have 


$$
\left\{\begin{array}{l}
\lambda_{K, k} \chi\left(\tilde{I}^{*}, \lambda\right)=\lambda^{*} \cdot \exp (Q[G, \Gamma] / n)^{*} \cdot V_{\widetilde{I}} \\
\pi_{K, k}\left(\lambda^{*} \cdot V_{a^{*}}\right)=\psi \alpha^{*} \in G \quad(n=[K: k], \psi=\psi(G, J(K / k)) .
\end{array}\right.
$$

Next we have

$$
\begin{aligned}
& \tau_{K, k}\left(u_{\sigma}\right)=f_{K \mid k}(G, \sigma)^{*}=\chi(\widetilde{\Gamma}(G, \sigma), \lambda(G, \sigma))^{*} \\
& \tau_{K, k}(\chi(\widetilde{\Gamma}, \lambda))=N_{G} \chi(\widetilde{\Gamma}, \lambda)=\chi\left(N_{G} \widetilde{I}, \lambda^{n} \cdot Q(G, \Gamma)\right)^{*} .
\end{aligned}
$$

Then using (4) and

$$
V \widetilde{\beta}_{\jmath}=\hat{\Gamma}(G, \sigma), \quad V(\pi \widetilde{\Gamma})=N_{G} \widetilde{\Gamma} \quad\left(V=V_{K \mid k}\right)
$$

we have

$$
\mu u_{\sigma}=\chi_{k}\left(\widetilde{\beta}_{\sigma}, \lambda(G, \sigma)\right), \mu \chi_{K}(\widetilde{\Gamma}, \lambda)=\chi_{k}\left(\pi \widetilde{\Gamma}, \lambda^{n} \cdot Q(G, \Gamma)\right)
$$

for $\quad \mu=\mu_{K, k}=\varphi_{K, k^{\circ}}^{-1} \lambda_{K, k}^{-1} \circ \tau_{K, k}$. Let $\psi=\psi(H(k), J(K / k))$ then for $\alpha^{*}=$ $\left(\pi \bar{\Gamma} \cdot \overline{\beta_{\sigma}}\right) \in J(K / k)$ we have

$$
\mu\left(\lambda^{*} \cdot U_{a^{*}}\right)=\chi_{k}\left(\psi \alpha^{*}, \lambda^{n} \cdot Q\left(G,\left[\alpha^{*}\right]\right)\right) .
$$

Finally from (18) follows that

$$
\mu_{K, k}\left(\lambda^{*} \cdot V_{a^{*}}\right)=\chi_{k}\left(\psi \alpha^{*}, \lambda^{n}\right) .
$$

Thus the homomorphisms $\lambda_{K, k}, \pi_{K, k}$ and $\mu_{K, k}$ are expressed by (21) and (22).

B. Let $G=\cup \bar{\sigma} H$, and for $\rho \in H$ let us take the same path on $R(K)$ for the extension $K / k$ and for $K / z$. Now put

$$
\begin{aligned}
& W(K / k)=\bigcup_{\sigma \in G} A(K)^{*} \cdot u_{\sigma}=\bigcup_{a^{*} J(K / k)} T^{*} \cdot V_{a^{*}} \\
& W(K / l)=\bigcup_{\rho \in H} A(K)^{*} u_{\rho}^{*} \underset{\beta^{*} \mathrm{e} J(K / \boldsymbol{t})}{\bigcup} T^{*} V_{\beta^{*}}^{*} .
\end{aligned}
$$

Then $\nu=\nu_{K} \geq, k$ is determined in the proof of Theorem 1. In the standard expression we have

$$
\left\{\begin{array}{l}
\nu_{K, z, k}\left(\lambda^{+}\right)=\lambda^{*} \quad(\lambda \in T) \\
\nu_{K, z, k}\left(V_{\beta^{*}}\right)=b_{K, \forall, k}\left(\beta^{*}\right) V_{\beta^{*}}
\end{array} \quad\left(\beta^{*} \in J(K / l)\right) .\right.
$$

Then for $m=[K: t]$ and $n=[l: k]$ we get

$$
b\left(\beta^{*}\right)=\exp \left(-Q_{K / t}\left[H,\left[\beta^{*}\right]\right] / m+Q_{K / k}\left[G,\left[\beta^{*}\right]\right] / m n\right) .
$$


Using the formula

$$
\left[\pi_{K / \ell} \Gamma,\left(\pi_{K / l} \mathfrak{P}\right)^{\bar{\sigma}^{-1}-1}\right]_{l}=\left[I^{\top}, N_{H} \mathfrak{P}^{-1}-1\right]_{K}
$$

we have

$$
b\left(\beta^{*}\right)=\exp \left(\left[\left[\beta^{*}\right],\left(N_{G} \mathfrak{P}\right)\left(N_{H} \mathfrak{P ^ { - n }}\right)\right]_{K} / m n\right)
$$

namely,

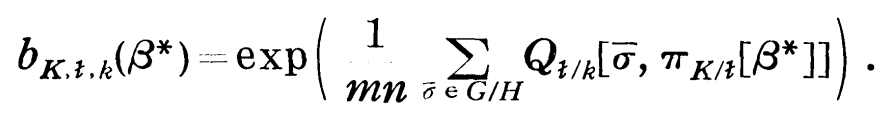

Thus the homomorphism $\nu_{K, \imath, k}$ is determened by (23) and (25) explicitly. C. Let $m=[L: K]$ and $n=[K: k]$. Let $G=\bigcup H \bar{\sigma}$. Using the results in $\mathrm{A}$ we can compute the term (25) in $\S 2$

$$
\begin{aligned}
& f_{L / k}(\sigma \cdot \tau)^{m}(\delta h)(\sigma, \tau)=\lambda_{L, K^{\circ}}^{-1} \tau_{L, K^{\circ}} \nu_{L, K, k}^{-1}\left(u_{\bar{\sigma}} u_{\bar{\tau}} u_{\bar{\sigma} \bar{\tau}}^{-1}\right) \\
& =\chi\left(N_{L} \widetilde{F}_{L / k}^{\prime}(\bar{\sigma}, \bar{\tau}), \lambda_{L / k}(\bar{\sigma}, \bar{\tau})^{m} \cdot Q_{L / k}\left(H, \Gamma_{L / k}^{\prime}(\bar{\sigma}, \bar{\tau})\right)\right.
\end{aligned}
$$

where we put $\Gamma_{L / k}^{\prime}(\bar{\sigma}, \bar{\tau})=B_{\bar{\sigma}} \cdot \bar{\sigma} B_{\bar{\tau}} \cdot B_{\frac{-1}{\sigma \tau}}$. On the other hand, let $B_{\sigma}$ $(\sigma \in G)$ be a fixed path from $\mathfrak{P}_{0}$ to $\sigma \mathfrak{P}_{0}$ on $R(L)$ and we take $\pi B_{\bar{\sigma}}=B_{\bar{\sigma}}$ on $R(K)\left(\pi=\pi_{L / K}\right)$. Then $\Gamma_{K / k}(\widetilde{\sigma}, \widetilde{\tau})$ on $R(K)$ is given by $\pi\left(B_{\bar{\sigma}}\left(\bar{\sigma} B_{\bar{\tau}}\right) B_{\overline{\sigma \tau}}^{-1}\right)$. Let us denote by $V$ the transfer mapping of $H(K)$ into $H(L)$ then we can see that

$$
V \tilde{\Gamma}_{K / k}(\widetilde{\sigma}, \widetilde{\tau})=N_{H} \widetilde{\Gamma}_{L / k}^{\prime}(\bar{\sigma}, \bar{\tau})
$$

holds. As in B, (24) we have

$$
\begin{aligned}
\lambda_{K / k}(\tilde{\sigma}, \tilde{\tau}) & =Q_{K / k}\left[\pi B_{\bar{\tau}},\left(\pi \mathfrak{P}^{\tau^{-1}-1}\right]=Q_{L / k}\left[B_{\bar{\tau}}, N_{H} \mathfrak{P}^{\bar{\sigma}^{-1}-1}\right]\right. \\
& =\lambda_{L / k}(\bar{\sigma} H, \bar{\tau}) \lambda_{L / k}(H, \bar{\tau})^{-1} .
\end{aligned}
$$

Furthermore, we have by (8)

$$
\begin{aligned}
& \lambda_{K / k}(\widetilde{\sigma}, \widetilde{\tau}) \cdot \lambda_{L / k}(\bar{\sigma}, \bar{\tau})^{-m} \cdot Q_{L / k}\left(H, \Gamma_{L / k}^{\prime}(\bar{\sigma}, \bar{\tau})\right)^{-1} \\
= & \left\{\lambda(\bar{\sigma} H, \bar{\tau}) \cdot \lambda(H, \bar{\tau})^{-1}\right\} \cdot \lambda(\bar{\sigma}, \bar{\tau})^{-m} \cdot\left\{\lambda(\bar{\sigma}, \bar{\tau})^{m} \cdot \lambda(H \bar{\sigma}, \bar{\tau})^{-1} \cdot \lambda(H, \overline{\sigma \tau}) \cdot \lambda(H, \bar{\sigma})^{-1}\right\} \\
= & \left(\delta g^{1}\right)(\widetilde{\sigma}, \widetilde{\tau})^{-1} \quad\left(\lambda=\lambda_{L / k}\right)
\end{aligned}
$$

where we put

$$
g_{K / k}^{1}(\widetilde{\sigma})=\lambda_{L / k}(H, \bar{\sigma})
$$

Then comparing these equalities we get

$$
\operatorname{infl}_{G, F^{\circ}} \boldsymbol{\varphi}_{L, K} f_{K / k}(\widetilde{\sigma}, \widetilde{\tau})\left(\delta g_{K / k}^{1}\right)(\widetilde{\sigma}, \widetilde{\tau})=f_{L / k}(\sigma, \tau)^{m} \bullet(\delta h)(\sigma, \tau)
$$


for the above normalization of $\Gamma_{K / k}(\widetilde{\sigma}, \widetilde{\tau})$. Now let

$$
\begin{aligned}
& W(L / k)=\bigcup_{\bar{\sigma}}\left\{\bigcup_{\rho \in H} A(L)^{*} u_{\rho}\right\} u_{\bar{\sigma}} \\
& W(K / k)=\bigcup_{\bar{\sigma} \in F} A(K)^{*} v_{\tilde{\sigma}}, \quad v_{\tilde{\sigma}}=g_{K / k}^{1}(\widetilde{\sigma}) \cdot u_{\tilde{\sigma}}
\end{aligned}
$$

so that

$$
v_{\tilde{\sigma}} v_{\tilde{\tau}}=f_{K / k}^{\prime}(\widetilde{\sigma}, \widetilde{\tau}) \cdot v_{\tilde{\sigma} \tilde{\tau}}, f_{K / k}^{\prime}(\widetilde{\sigma}, \widetilde{\tau})=f_{K / k}(\widetilde{\sigma}, \widetilde{\tau}) \cdot\left(\delta g_{K / k}^{1}\right)(\widetilde{\sigma}, \widetilde{\tau}) .
$$

Then we can apply the results in (iv) in the proof of Theorem 1 for these expressions. Namely, $\rho=\rho_{L, K, k}$ is given by

$$
\left\{\begin{array}{l}
\rho=\lambda_{K, k^{\circ}} \mu_{L, K^{\circ}} \nu_{L, K, k}^{-1} \quad \text { on } \nu_{L, K, k} W(L / K) \\
\rho\left(u_{\bar{\sigma}}\right)=v_{\tilde{\sigma}}
\end{array}\right.
$$

Then we shall use the standard expressions of $W(L / k)$ and $W(K / k)$ :

$$
W(L / k)=\underset{a^{*} \in J(L / k)}{\bigcup} T^{*} \cdot V_{a^{*}}, W(K / k)=\bigcup_{a^{*} \in J(K / k)} T^{*} \cdot V_{a^{*}}^{*} \cdot
$$

We can see first that $\lambda^{*} \in T^{*}$ is mapped by $\rho$ to $\left(\lambda^{m}\right)^{*}$. For $\beta^{*}=\beta_{\frac{\sigma}{\sigma}}^{*}$ $V_{\beta^{*}}=\exp \left(-Q_{L / k}\left[G, B_{\bar{\sigma}}\right] / m n\right)^{*} \cdot u_{\sigma}$ is mapped by $\rho$ to $\exp \left(-Q_{L / k}[G\right.$, $\left.\left.B_{\bar{\sigma}}\right] / n\right)^{*} \cdot v_{\tilde{\sigma}}=\exp \left(\left\{Q_{K / k}\left[F, \pi B_{\bar{\sigma}}\right]-Q_{L / k}\left[G, B_{\bar{\sigma}}\right]\right\} / n\right)^{*} \cdot \lambda_{L / k}(H, \bar{\sigma})^{*} \cdot V_{\tilde{\sigma}}$. Here the component in $T^{*}$ is 1 which can be proved similarly as in $B$. Hence we have $\rho\left(V_{\beta^{*}}\right)=V_{\psi \beta^{*}}^{*}$ for $\beta^{*}=\beta_{\bar{\sigma}}^{*}$ (where $\psi=\psi(J(K / k), J(L / k))$. Вy similar computations we also get that $\rho\left(V_{a^{*}}\right)=V_{\psi^{*} a^{*}}^{*}$ holds for $\alpha^{*}=$ $\pi \bar{\Gamma}^{*} \cdot \beta^{*}$. Therefore the homomorphism $\rho_{L, K, k}$ is given for the standard expression by

$$
\begin{cases}\rho_{L, K, k}\left(\lambda^{*}\right)=\left(\lambda^{m}\right)^{*} & (\lambda \in T) \\ \rho_{L, K, k}\left(V_{a^{*}}\right)=V_{\psi a^{*}}^{*} & \left(\alpha^{*} \in J(K / k)\right)\end{cases}
$$

with the above normalization of $\Gamma_{K / k}(\widetilde{\sigma}, \widetilde{\tau})$.

REMARK. If we normalize $\left[\alpha^{*}\right]$ on $R(L)$ and $\left[\psi \alpha^{*}\right]$ on $R(K)$ to be $\left[\psi \alpha^{*}\right]=\pi_{L / K}\left[\alpha^{*}\right]$ then the 2 -cocycle of the group extensions are

$$
\begin{aligned}
& b_{L / k}\left(\alpha^{*}, \beta^{*}\right)=\exp \left(\frac{2 \pi i}{m n} \sum_{\tau \in G} \operatorname{LK}\left(\left[\alpha^{*}\right]+\left(\psi^{\prime} \alpha^{*}\right)\left[\beta^{*}\right]-\left[\alpha^{*} \beta^{*}\right], \tau \Re\right)\right) \\
& b_{K / k}\left(\psi \alpha^{*}, \psi \beta^{*}\right)=\exp \left(\frac { 2 \pi i } { n } \sum _ { \tau \in F } \operatorname { L K } \left(\pi\left[\alpha^{*}\right]+\left(\psi^{\prime} \psi \alpha^{*}\right) \pi\left[\beta^{*}\right]\right.\right.
\end{aligned}
$$




$$
\left.\left.-\pi\left[\alpha^{*} \beta^{*}\right], \widetilde{\tau} \pi \mathfrak{P}\right)\right)
$$

By (27) these must satisfy the relation

$$
b_{L / k}\left(\alpha^{*}, \beta^{*}\right)^{m}=b_{K / k}\left(\psi \alpha^{*}, \psi \beta^{*}\right) \text {. }
$$

But we can also verify (28) dirsctly from the above expressions of $b_{L / k}\left(\alpha^{*}, \beta^{*}\right)$ and $b_{K / k}\left(\psi \alpha^{*}, \psi \beta^{*}\right)$ using the formula $\operatorname{LK}\left(\pi_{L / K} \Gamma, \pi_{L / K} \mathfrak{P}\right)_{K}$ $=\sum_{\rho \in H} \operatorname{LK}\left(I^{\prime}, \rho \mathfrak{P}\right)_{L}$.

$\mathrm{A}^{\prime}$. Now we have to check the formulas in $A^{\prime}$. As we have seen already (Kawada-Tate [8], or Kawada [7]) the generalized normresidue symbol $(\chi, k)$ for $\chi=\chi(\widetilde{\gamma}, \lambda) \in A(k)$ can be identified with $\sigma(\tilde{\gamma})$. Here $\sigma(\bar{\gamma})$ means the automorphism of $A_{k} / k$ which is attached canonically to $\gamma$. Also we can identify each $\gamma \in F(k)$ with the automorphism of $\Omega / k$ which is induced canonically by $\gamma$ in the classical theory. Then we can verify that the homomorphism $\xi_{K, k}$ is given by

$$
\xi_{K, k}\left(\lambda^{*} \cdot V_{a^{*}}\right)=\alpha^{*} \quad(\in J(K / k))
$$

for which all the formulas in $A^{\prime}$ are satisfied.

\section{$\S 5$. System of generalized Weil groups in algebraic function fields}

1. We shall consider in this section the system of generalized Weil groups in algebraic function field. If we could apply the general method in $\S 3$ we should have rather too big groups $\{W(k)\}(k \in \Re)$. Hence we shall choose a suitable subgroup of each $W(k)$ which satisfy the properties in Theorems 4,5 in $\S 3$ with suitable modifications.

THEOREM 6. In the class formation $\{\Omega, A(K)\}$ defined in $\S 4,1$ in algebraic function fields there exists a system of generalized Weil groups $\{W(k)\}(k \in \Re)$ which satisfy the following properties:

$\left(\mathrm{A}_{1}\right)^{*}$ There exist

an onto-homomorphism $\pi_{k}: W(k) \rightarrow F(k)$

an into-isomorphism $\lambda_{k}: R \rightarrow W(k)$

an onto-homomorphism $\mu_{k}: W(k) \rightarrow E(k)^{\wedge}$

such that 


$$
\begin{aligned}
& 0 \longrightarrow R \stackrel{\lambda_{k}}{\longrightarrow} W(k) \stackrel{\pi_{k}}{\longrightarrow} F(k) \longrightarrow 0 \\
& 1 \longrightarrow W(k)^{\iota} \stackrel{\iota}{\longrightarrow} W(k) \stackrel{\mu_{k}}{\longrightarrow} E(k)^{\wedge} \longrightarrow 0
\end{aligned}
$$

are exact sequences. Here $\lambda_{k} R$ is contained in the centre of $W(k)$. Let $\varepsilon: a \rightarrow \exp (2 \pi i a)(a \in R)$. The following diagram is also commutative, where each column and each row are exact sequences:

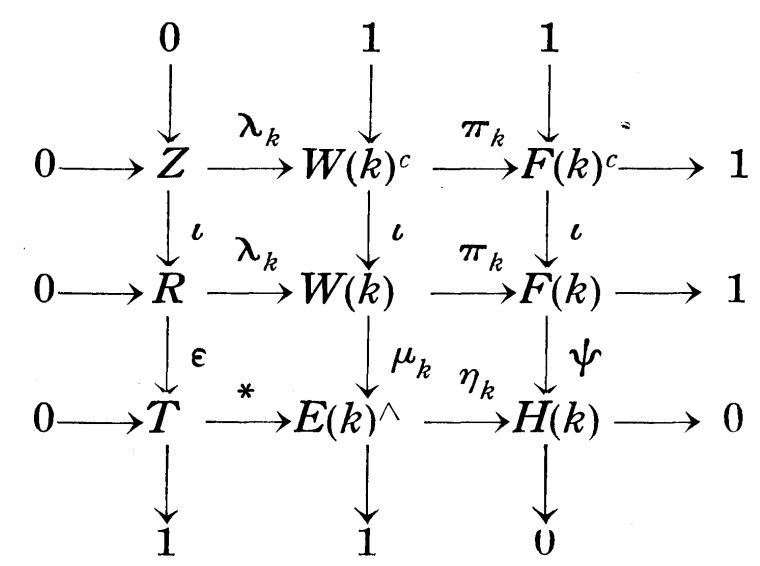

$\left(\mathrm{B}_{1}\right)^{*} \quad$ Let $k \subset l(k, \imath \in \Omega)$ and $m=[l: k]$. Then there exists

$$
\text { an into-isomorphism } \quad \nu_{\ell, k} W(\mathfrak{l}) \rightarrow W(k)
$$

such that

(4)

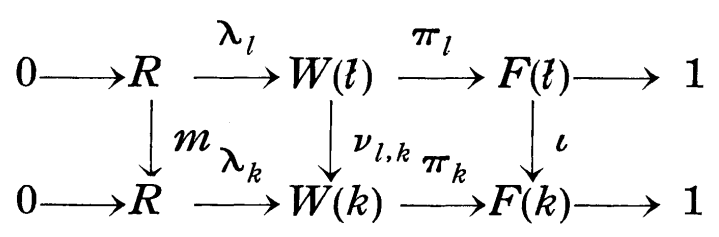

is a commutative diagram, where $m$ denotes the homomorphism $a \rightarrow m a$ $(a \in R)$.

$\left(\mathrm{C}_{1}\right)^{*}$ For normal $K / k$ there exists

$$
\text { an onto-homomorphism } \rho_{K, k}: W(k) \rightarrow W(K / k)
$$

such that 
(6)

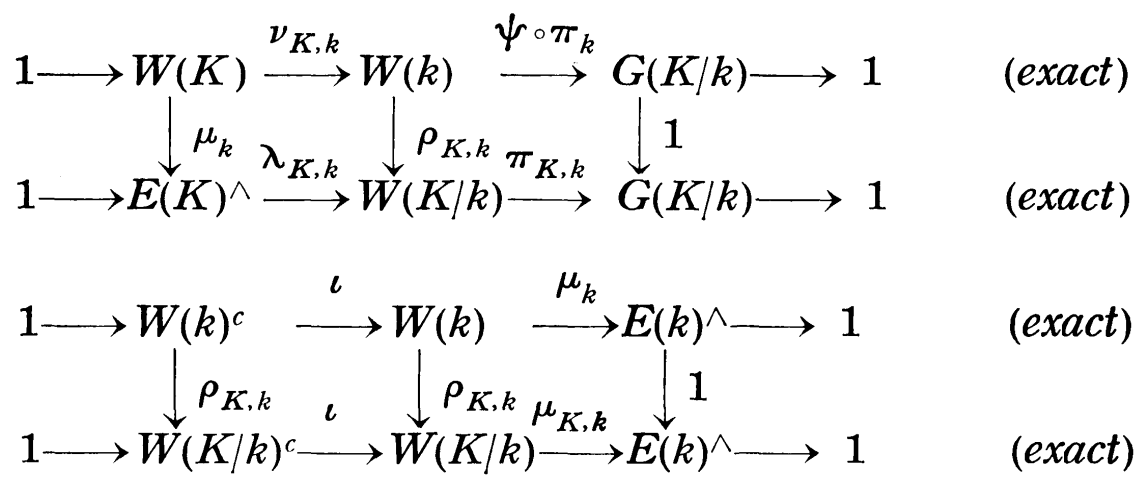

are commutative diagrams. Moreover, the following diagram is also commutative, where each column and each row are exact:

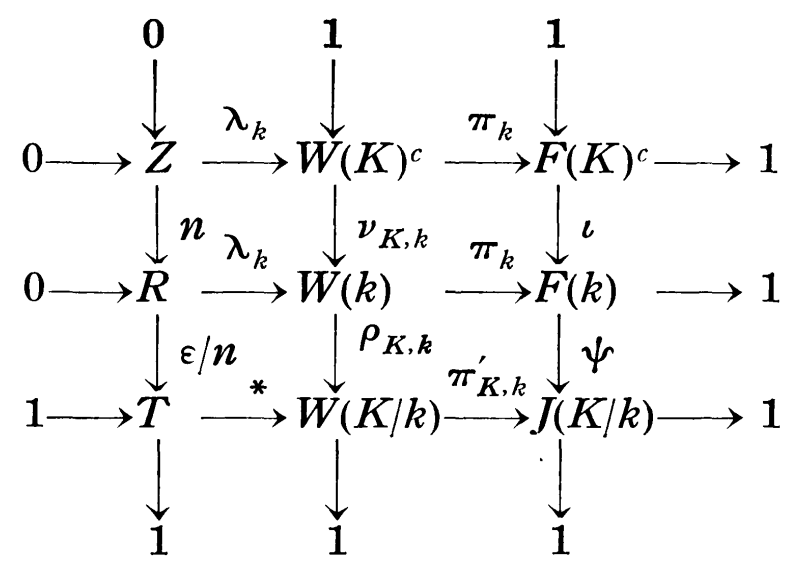

where $n=[K: k]$.

(PROOF) (i) We shall define $W(k)$ directly as a group extension of $R$ by $F(k)$. Let $\left\{\alpha_{1}, \cdots, \alpha_{g}, \beta_{1}, \cdots, \beta_{g}\right\}$ be a system of generators of $F(k)$ and $\prod_{j} \alpha_{j} \bullet \beta_{j} \cdot \alpha_{j}^{-1} \bullet \beta_{j}^{-1}$ be the fundamental relation among them, where $g$ denotes the genus of $R(k)$. Let $\mathfrak{F}_{k}$ be the free group generated by the free generators $\left\{A_{1}, \cdots, A_{g}, B_{1}, \cdots, B_{g}\right\}$. Put $C=$ $\prod_{j} A_{j} B_{j} A_{j}^{-1} B_{j}^{-1}$ and let $\mathfrak{N}(C)$ be the normal subgroup of $\mathfrak{F}_{k}$ generated by $C$. Then by the mapping $A_{j} \rightarrow \alpha_{j}, B_{j} \rightarrow \beta_{j}$ we have $\mathfrak{F}_{k} / \mathfrak{N}(C) \cong F(k)$. Let us fix a representative $W(\alpha) \in \mathfrak{F}_{k}$ for each $\alpha \in F(k)$ in the corresponding class. In particular, we choose $W\left(\alpha_{i}\right)=A_{i}, W\left(\beta_{i}\right)=B_{i}$ $(i=1,2, \cdots, g)$. For each pair $(\alpha, \beta)$ we have

$$
W(\alpha) \cdot W(\beta) \cdot W(\alpha \beta)^{-1}=\prod_{i} T_{i} \cdot C^{\varepsilon_{i}} \cdot T_{i}^{-1} \in \mathfrak{N}(C) \quad\left(\varepsilon_{i}= \pm 1\right)
$$

Then we define 


$$
a_{k}(\alpha, \beta)=\sum_{i} \varepsilon_{i} \quad \in Z \quad(\subset R)
$$

Clearly $a_{\dot{k}}(\alpha, \beta)$ is a 2-cocycle of $F(k)$ over $Z$ (or over $R$ ). Hence we can define the group $W(k)$ by

$$
\left\{\begin{array}{l}
W(k)=\bigcup_{a \in F} R^{*} \cdot V_{a} \quad\left(R^{*}=\left\{\lambda^{*} ; \lambda \in R\right\}\right) \\
V_{\alpha} \cdot V_{\beta}=a_{k}(\alpha, \beta)^{*} \cdot V_{\alpha}
\end{array}\right.
$$

where $R^{*}$ belongs to the centre of $W(k)$. Let us define then

$$
\left\{\begin{array}{l}
\lambda_{k}(\lambda)=\lambda^{*} \quad(\lambda \in R) \\
\pi_{k}\left(\lambda^{*} \cdot V_{a}\right)=\alpha \quad(\alpha \in F(k)) \\
\mu_{k}\left(\lambda^{*} \cdot V_{a}\right)=\chi(\tilde{\alpha}, \exp (2 \pi i \lambda)) \in E(k)^{\wedge}
\end{array}\right.
$$

where $\tilde{\alpha}$ means the class of $\alpha \in F(k) \bmod F(k)^{c}$. Then

$$
\text { Kernel } \mu_{k}=\bigcup_{r \in F(k)^{c}} Z^{*} \cdot V_{r}
$$

It is evident that $W(k)^{c}$ is included in Kernel $\mu_{k^{*}}$. To see the converse it is enough to prove $Z^{*} \subset W(k)^{c}$. This follows from $1^{*}=\prod \operatorname{I} V_{a_{i}} \cdot V_{\beta_{i}}$ • $V_{a_{i}}^{-1} \cdot V_{\beta_{i}}^{-1} \in W(k)^{c}$, which is a consequence of $\prod_{j} W\left(\alpha_{j}\right) \cdot W\left(\beta_{j}\right) \cdot W\left(\alpha_{j}\right)^{-1}$. $W\left(\beta_{j}\right)^{-1}=C$ in $\mathfrak{F}_{k^{*}}$. The commutativity of the diagram (3) follows from the above results.

(ii) B. Let us identify $F(l)$ with the subgroup $\pi F(t) \subset F(k)$. Take the same element $W(\beta)$ for $\beta \in F(t)$ both in $\mathfrak{F}_{z}$ and in $\mathfrak{F}_{k}$, and $[\beta]$ be the path corresponding to $W(\beta)$. Let $F(k)=\bigcup \bar{\sigma} \cdot F(\mathfrak{l})$ and for $\beta \in F(\mathfrak{l})$ let us put

$$
b_{z / k}(\beta)=\left[[\beta], \prod_{\bar{\sigma}} \mathfrak{P}^{\bar{\sigma}^{-1}} \cdot \mathfrak{P}^{-m}\right]_{l} /(2 \pi i)
$$

Then for

$$
W(k)=\bigcup_{a \in F, k)} R^{*} \cdot V_{a}, \quad W(l)=\bigcup_{\beta \in F(z)} R^{\dagger} \cdot V_{\beta}^{\prime \cdot}
$$

let us define

$$
\left\{\begin{array}{l}
\nu_{\imath k}\left(\lambda^{*}\right)=(m \lambda)^{*} \quad(\lambda \in R) \\
\nu_{z k}\left(V_{\beta}^{\dagger \cdot}\right)=b_{z / k}(\beta) \cdot V_{\beta} .
\end{array}\right.
$$

From the relations $b\left(\beta \cdot \beta^{\prime}\right)=b(\beta) \cdot b\left(\beta^{\prime}\right)$ and $a_{k}(\beta, \gamma)=m a_{t}(\beta, \gamma)\left(\beta, \beta^{\prime}\right.$, 
$\gamma \in F(t)$ ) follows that $\nu$ is a homomorphism and the diagram (4) is commutative. We can also verify (5) easily.

(iii) For normal $K / k$ let

$$
W(k)=\bigcup_{a \in F(k)} R^{*} \cdot V_{a}, \quad W(K / k)=\bigcup_{a^{*} \in J(K / k)} T^{*} \cdot V_{a}^{*}
$$

To each $\alpha^{*} \in J(K / k)$ let us fix a representative $\alpha \in F(k)$ (i. e. $\alpha^{*}=\alpha$ $\bmod F(K)^{c}$ ) and let $\left[\alpha^{*}\right]$ be a closed path on $R(k)$ which corresponds to $W(\alpha) \in \mathfrak{F}_{k^{*}}$. Then we define the homomorphism $\rho$ by

$$
\left\{\begin{array}{l}
\rho_{K, k}\left(\lambda^{*}\right)=\exp (2 \pi i \lambda / n) \quad(\lambda \in R) \\
\rho_{K, k}\left(V_{a}\right)=V_{a^{*}}^{*}
\end{array}\right.
$$

Then from the definition of $b_{K / k}$ in $\S 4$ follows that

$$
b_{K / k}\left(\alpha^{*}, \beta^{*}\right)=\exp \left(2 \pi i a_{k}(\alpha, \beta) / n\right)
$$

We can verify the commutativity of diagrams (6), (7), (8) easily, q.e.d.

2. Here we shall view the group $W(k)$ from another point of view. For that purpose let us define the groups $W^{*}(k)$ and $W^{*}(K / k)$ by

$(20)$

$$
\left\{\begin{array}{l}
W^{*}(k)=\underset{a \in F(k)}{\bigcup} Z^{*} \cdot V_{a} \\
V_{a} \cdot V_{\beta}=a_{k}(\alpha, \beta) \cdot V_{a \beta}, \lambda^{*} \cdot V_{a}=V_{a} \cdot \lambda^{*} \quad(\lambda \in Z) \\
a_{k}(\alpha, \beta) \text { as in (11) }
\end{array}\right.
$$

$$
\left\{\begin{array}{l}
W^{*}(K / k)=\bigcup_{a^{*} \in J(K / k)}(Z / n Z)^{*} \cdot V_{a^{*}}^{*} \\
V_{a^{*}}^{*} \cdot V_{\beta^{*}}^{*}=c_{K / k}\left(\alpha^{*}, \beta^{*}\right)^{*} \cdot V_{a^{*} \beta^{*}} \\
c_{K / k}\left(\alpha^{*}, \beta^{*}\right)=\sum_{\tau \in G} \operatorname{LK}\left(\left[\alpha^{*}\right]+\left(\psi^{\prime} \alpha^{*}\right)\left[\beta^{*}\right]-\left[\alpha^{*} \beta^{*}\right], \tau \Re\right)(\bmod n Z)
\end{array}\right.
$$

Let us denote

$$
M^{*}(K / k)=\bigcup_{a^{*} \mathrm{e} H(K)}(Z / n Z)^{*} \cdot V_{a}^{*} \quad\left(\subset W^{*}(K / k)\right) .
$$

Since $c_{K / k}\left(\alpha^{*}, \beta^{*}\right) \equiv 0(\bmod n)$ for $\alpha^{*}, \beta^{*} \in H(K) M^{*}(K / k)$ is a split extension of $(Z / n Z)$ by $H(K)$.

Now let us fix a point $\mathfrak{p}$ on the Riemann surface $R(k)$ and let $R^{*}(k)=R(k)-\{\mathfrak{p}\}$. Let $C$ be a small circle around $\mathfrak{p}$ in the negative 
sense. Then the fundamental group (S) of $R^{*}(K)$ is a free group with $2 \mathrm{~g}$ free generators $\left\{A_{1}, \cdots, A_{g} . B_{1}, \cdots, B_{g}\right\}$ such that $C=\prod_{j} A_{j} B_{j}$ $A_{j}^{-1} B_{j}^{-1}$. Consider $\mathfrak{N}(C)^{c} \subset \mathfrak{N}(C) \subset \mathbb{S}$ then from the definition of the 2-cocycle $a_{k}(\alpha, \beta)$ and from (20) follows that

$$
W^{*}(k) \cong\left(\mathfrak{S} / \mathfrak{N}(C)^{c}\right. \text {. }
$$

For, $a_{k}(\alpha, \beta)$ is none other than the 2-cocycle of the group extension of $\mathfrak{R}(C) / \mathfrak{N}(C)^{\complement} \cong Z$ by $F(k)$.

Let $K / k$ be a finite unramified extension with $G=G(K / k)$ and let $R^{*}(K / k)=R(K)-\{\tau \mathfrak{P} ; \tau \in G\}$ where $\pi \mathfrak{P}=\mathfrak{p}$. Since $R^{*}(K / k)$ is an unramified regular covering of $R^{*}(k)$ there exists a normal subgroup $\mathfrak{N}_{K / k}$ of (S) such that

$$
\left(\mathfrak{S} / \mathfrak{N}_{K / k} \cong G(K / k)\right.
$$

Let us identify $F(K)$ with $\pi F(K) \subset F(k)$ and for $\alpha \in F(K)$ let us take the same element $W(\alpha)$ both in $\mathfrak{F}_{K}$ and in $\mathfrak{F}_{k^{*}}$. In the isomorphism (23) we have

$$
\Re_{K / k} / \mathfrak{N}(C)^{c} \cong \bigcup_{\gamma \in F(K)} Z \cdot V_{\gamma}
$$

and we may identify this group with $W^{*}(K)$. Now for $\alpha^{*} \in J(K / k)$ let us fix a representative $\alpha \in F(k)$ in its class and take $\left[\alpha^{*}\right]=W(\alpha)$ on $R^{*}(K / k)$. Then let us define the homomorphism $\rho_{K, k}^{*}: W^{*}(k) \rightarrow$ $W^{*}(K / k)$ by

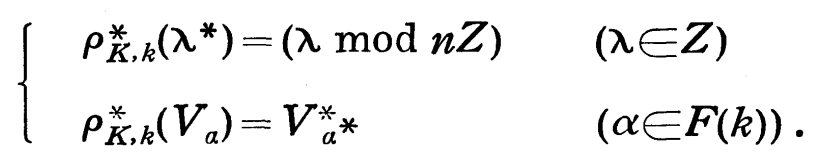

$\rho_{K, k}^{*}$ is an onto-homomorphism and

$$
\text { Kernel } \rho_{K / k}^{*}=\bigcup_{\gamma \in F(K)^{c}}(n Z)^{*} \cdot V_{r}=W^{*}(K)^{c} .
$$

Thus we have proved:

THEOREM 7. (i) Let (S) be a free group with $2 \mathrm{~g}$ free generators. There exists a normal subgroup $\mathfrak{N}_{k}(=\mathfrak{N}(C)$ ) such that

$$
\left(\mathfrak{S} / \mathfrak{R}_{k} \cong F(k), \dot{\mathfrak{S}} / \mathfrak{N}_{k}^{c} \cong W^{*}(k), \mathfrak{N}_{k} / \mathfrak{N}_{k}^{c} \cong Z .\right.
$$

(ii) For normal $K / k$ there exists a normal subgroup $\mathfrak{N}_{K / k}$ of (S) such 
that

$$
\left(\mathfrak{S} / \mathfrak{N}_{K / k} \cong G(K / k), \mathfrak{S} / \mathfrak{N}_{K / k}^{c} \cong W^{*}(K / k), \mathfrak{N}_{K / k} / \mathfrak{N}_{K / k}^{c} \cong M^{*}(K / k)\right.
$$

(iii) If we consider (S) as the fundamental group of $R^{*}(k)$ then $\mathfrak{N}_{k}$ and $\mathfrak{N}_{K / k}$ are the to unramified extensions $\Omega$ and $K$ corresponding subgroups respectively. The homomorphism. $\rho_{K, k}^{*}$ is defined by the canonical homomorphism between these groups.

\section{University of Tokyo}

\section{Bibliography}

[1] Y. Akizuki, Eine homomorphe Zuordnung der Elemente der Galoisschen Gruppe zu den Elementen einer Untergruppe der Normklassengruppe, Math. Ann., 112 (1936), 566-571.

[2] E. Artin, Algebraic numbers and algebraic functions I, Mimeographed Notes, New York University, (1951).

[3] E. Artin and J. Tate, Algebraic numbers and algebraic functions II, Mimeographed Notes (in preparation).

[4] C. Chevalley, Class field theory, Nagoya University (1953-54).

[5] G. Hochschild and T. Nakayama, Cohomology in class field theory, Ann. of Math., 55 (1952), 348-366.

[6] Y. Kawada, On the structure of the Galois group of some infinite extensions II, J Fac. Sci. Tokyo Univ., 7 (1954), 87-106.

[7] Y. Kawada, Class formations, Duke Math. J., 22 (1955), 165-178.

[8] Y. Kawada and J. Tate, On the Galois cohomology of unramified extensions of function fields in one variable, Amer. J. Math., 77 (1955), 197-217.

[9] Y. Kawada and I. Satake, Class formations II, J. Fac. Sci. Univ. Tokyo, 7 (1955), 353-389.

[10] T. Nakayama, Idèle class factor sets and class field theory, Ann. of Math., 55 (1952), 73-84.

[11] J. Tate, The higher dimensional cohomology groups of class field theory, Ann. of Math., 56 (1952), 294-297.

[12] A. Weil, Sur la théorie du corps de classes, This Journal, 3 (1951), 1-35.

[13] H. Weyl, Die Idee der Riemannschen Fläche, Leipzig, 2. Auf. (1923) 